\title{
The chirality of Yariv reagent aggregates correlates with AGP-binding ability
}

Raghuraj Hoshing, Blaise W Leeber III, Helene Kuhn, David Caianiello, Brandon Dale, Michael Saladino, Robert Lusi, Natalie Palaychuk, Sarah Weingarten, and Amit Basu*

Department of Chemistry, Brown University, Providence RI, USA 02912

abasu@brown.edu

\begin{abstract}
Yariv reagents are glycosylated triphenylazo dyes, some of which bind to the polysaccharide component of arabinogalactan proteins (AGPs), proteoglycans found in plant cell walls. However, the exact reason for the selectivity in the presence/absence of AGP binding ability among Yarivs remains unknown. The Yariv reagents are known to form supramolecular aggregates in solution. We use circular dichroism to show that the Yariv reagent aggregates possess helical chirality, and the AGP binding ability of the Yariv reagents is correlated to its helical chirality.
\end{abstract}


The Yariv reagents are a set of glycosylated triphenyl azo dyes (Figure 1), some of which bind plant proteoglycans called arabinogalactan proteins (AGPs). AGPs are ubiquitous components of plant cell walls found in numerous tissues and species and are implicated in a variety of cell signaling roles. ${ }^{1-3}$ The Yariv reagents are utilized to detect the presence of AGPs in plant cell wall fractions, to isolate AGPs, to stain AGPs for microscopy and, and in cellular assays to elucidate the role of AGPs by disrupting their physiological activities..$^{4-5}$ Despite the fact that the Yariv reagents are the most commonly used tool to study AGPs, the precise nature of interaction between AGPs and the Yariv reagents is not understood. The carbohydrate portion of AGPs that bind Yariv reagents contain a 1,3- $\beta$-D-galactan main chain and an arabinosylated 1,6$\beta$-D-linked galactan side chain. A series of biochemical and chemical degradation studies have identified the core 1,3- $\beta$-D-galactan main chain, which is proposed to adopt a helical conformation, as the Yariv reagent binding epitope in AGPs. ${ }^{6,7}$ The AGP-binding ability of the Yarivs reagents is dependent on the identity of the carbohydrate residue present in a given Yariv reagent. With the exception of $\beta$-D-mannosyl Yariv reagent 8, those Yariv reagents that terminate in $\beta$-D-linked sugars typically bind AGPs, whereas those terminating in $\beta$-L-, $\alpha$-D-, or $\alpha$-L- sugars do not bind AGPs. ${ }^{8}$ The basis for this carbohydrate-dependent selectivity has remained unclear to date. 

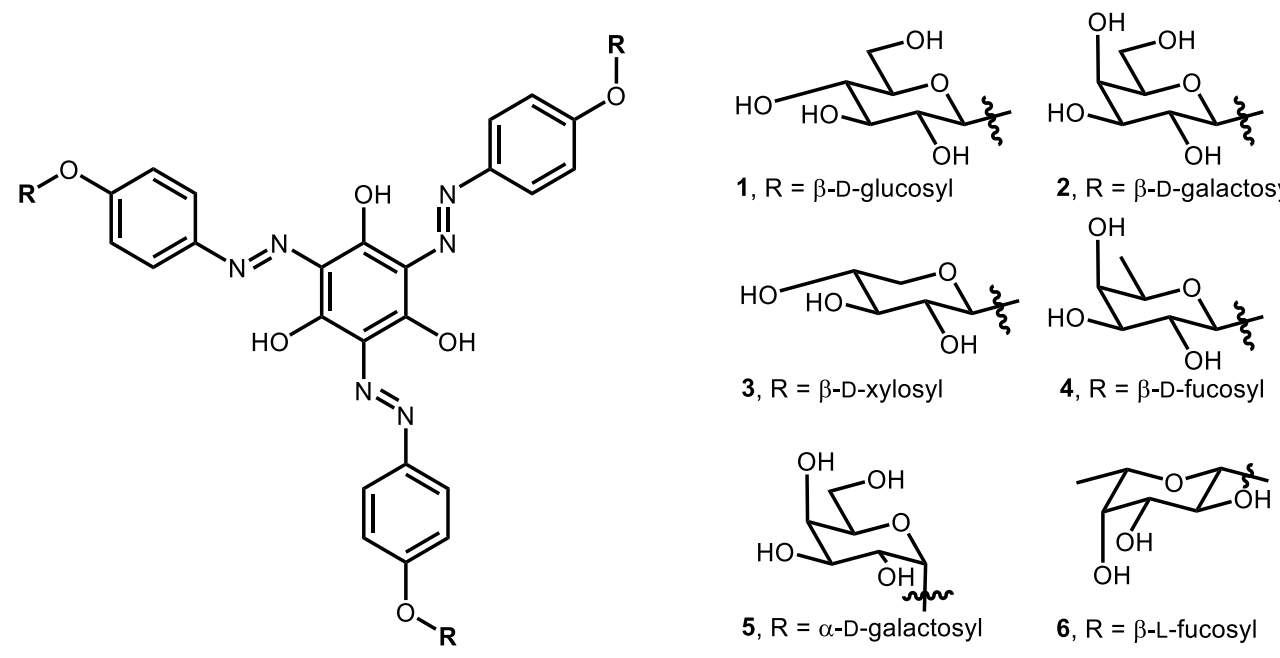

3, $R=\beta$-D-xylosyl
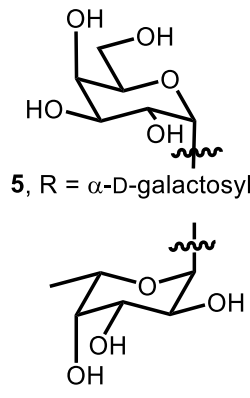

7, $R=\alpha-L$-fucosyl

2, $R=\beta$-D-galactosyl

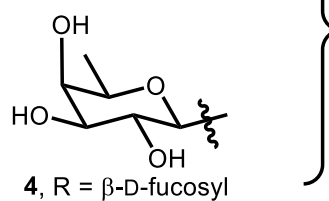

Bind AGP
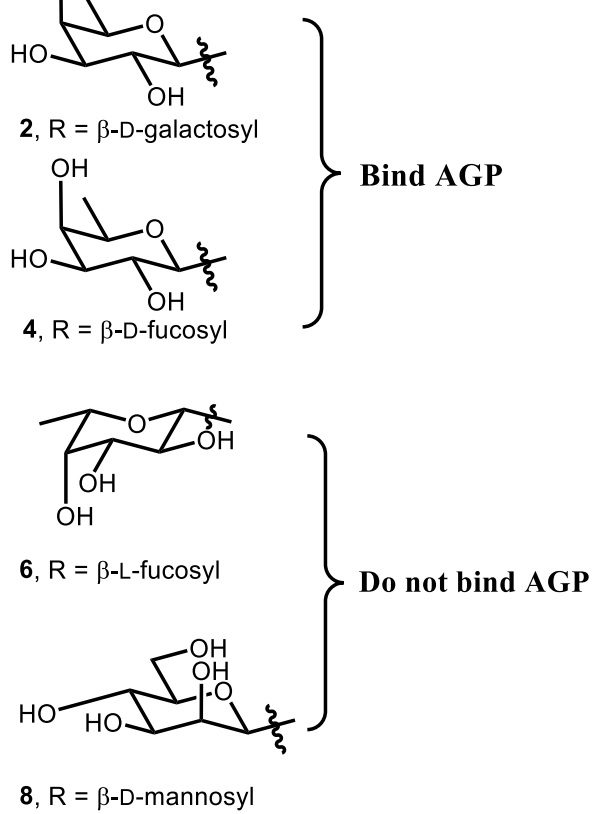

Figure 1. Yariv reagent structures

Previous studies using analytical ultracentrifugation (AUC) and NMR spectroscopy have shown that Yariv reagents self-aggregate in aqueous solution. ${ }^{7,}$ 9-10 The Yariv reagents are structurally similar to several other glycoconjugates that contain an aromatic core and peripheral glycosyl moieties, which form well characterized helical aggregates in solution. ${ }^{11-20}$ Based upon the finding that the core binding epitope for the Yariv reagents is a helical polysaccharide, we hypothesized that the Yariv reagents form helical aggregates. In this work we report the circular dichroism (CD) spectra of various Yariv reagents. We demonstrate that all Yariv reagents that bind AGP also exhibit bisignate exciton couplets under aggregating conditions, which we postulate arise from the formation of helical aggregates. Our results provide a basis for explaining the selectivity of AGP binding amongst the various Yariv reagents. 


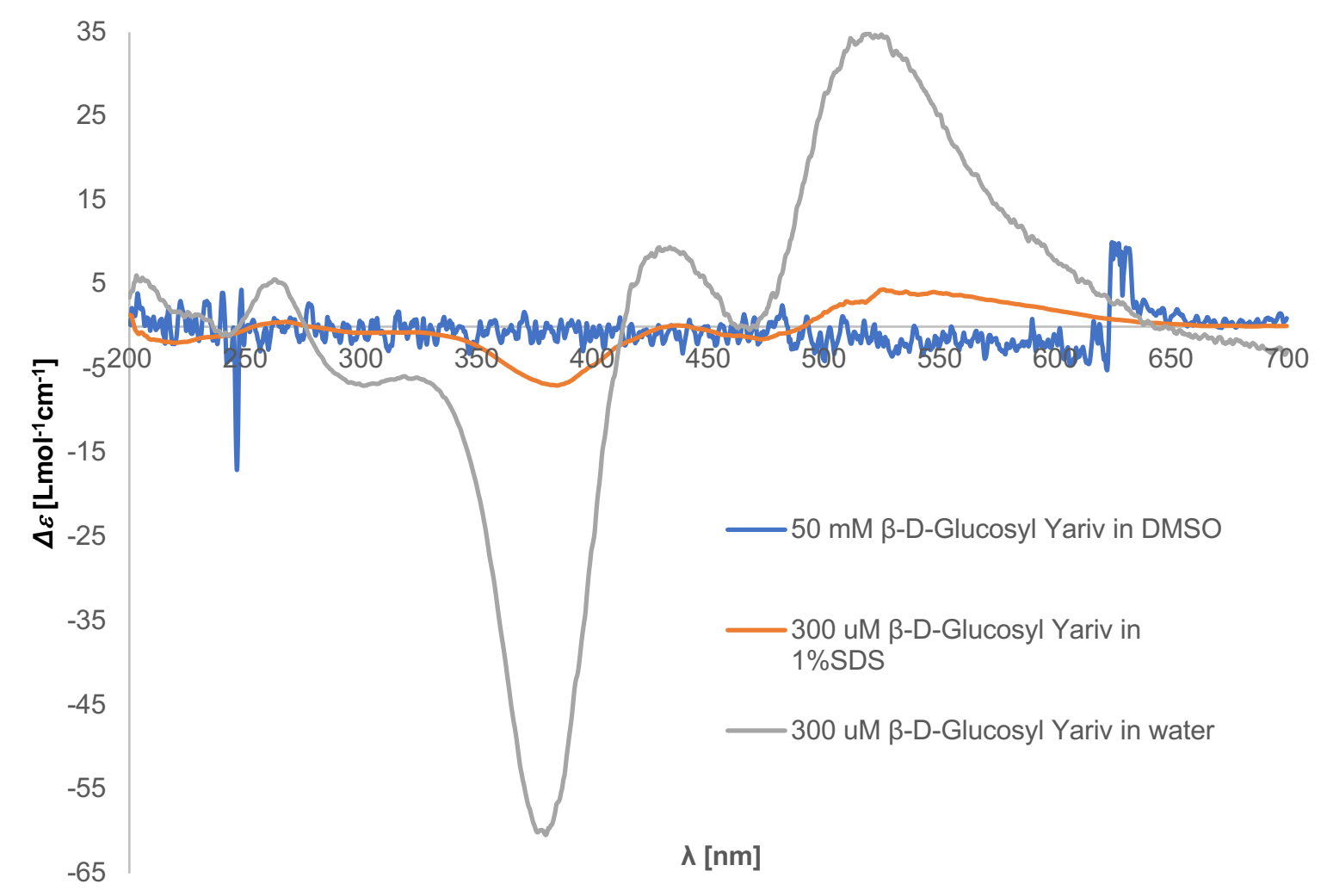

Figure 2. Comparison of CD spectra of $\beta$-D-Glucosyl Yariv (1) in aggregating conditions (water) and in disaggregating conditions (DMSO and SDS)

The Yariv reagents 1-7 were synthesized using reported procedures ${ }^{21}$, and 8 was purchased from Biosupplies, Australia. The CD spectrum of $\mathbf{1}$ in water, where the Yariv reagent is known to aggregate, shows the presence of a strong CD signal (Figure 2). The shape of the CD signal in water (Figure 2) indicates the presence of two overlapping bands, each of which exhibits a bisignate Cotton effect $\left(\lambda=380 \mathrm{~nm}, \Delta \varepsilon=-60 \mathrm{~L}^{\prime} \mathrm{mol}^{-1} \mathrm{~cm}^{-1} ; \lambda=527 \mathrm{~nm}, \Delta \varepsilon=34 \mathrm{~L}^{\prime} \cdot \mathrm{mol}^{-1} \mathrm{~cm}^{-1}\right)$. The two exciton-coupling induced bisignate Cotton effects present in the $\mathrm{CD}$ spectra arise from two distinct chromophores that are visible in the UV/vis spectrum, which maxima at $398 \mathrm{~nm}$ and 487 $\mathrm{nm}$, where the former has a higher absorbance (Figure S1). The exact identity of the chromophores that give rise to these peaks in these tris azo-dyes has not been unambiguously established and is the subject of on-going studies. ${ }^{7,22-24}$ Nonetheless, the presence of bisignate Cotton effects 
provides clear evidence that $\mathbf{1}$ forms chiral aggregates in water. Although assigning the exact handedness of the aggregates will require further knowledge of the morphology of the aggregates ${ }^{25}$ ( $\mathrm{H}$ vs J), comparison of the Yariv reagent structure to other C3-symmteric molecules suggests that the Yariv reagent aggregates are likely $\mathrm{H}-\mathrm{yype}^{26,27}$ (stacking). This would suggest that the positive sign of both couplets indicates the formation of right-handed helical aggregates. Further characterization of the Yariv aggregates to confirm this analysis is also the focus of on-going studies.

Most importantly, three critical pieces of evidence support the conclusion that the bisignate Cotton effects arise from the aggregate and are not an intrinsic property of the monomer. First, increasing temperature decreases the $\mathrm{CD}$ signal, consistent with decreased aggregation at higher temperatures (Figure S3). Second, the CD spectra of $\mathbf{1}$ was also acquired in the presence of sodium dodecyl sulfate (SDS) and DMSO (Figure 2). The presence of SDS reduce aggregations, as evidenced by decrease in the aggregate size by AUC, accompanied by a shift in the higher absorbance from the $398 \mathrm{~nm}$ peak to the longer wavelength $487 \mathrm{~nm}$ peak. This change in the $\mathrm{UV} /$ vis spectrum is also observed when the spectrum of $\mathbf{1}$ is obtained in DMSO (Figure S2), and the NMR spectrum of 1 in DMSO- $d_{6}$ also shows clearly resolved sharp resonances with none of the broadening associated with aggregation. ${ }^{7,21}$ As seen in Figure 2, the CD signal is reduced greatly in intensity in the presence of SDS, while in DMSO the CD signal is flat. Finally, we have prepared an analog of $\mathbf{2}$ containing a single azo-linked galactoside (S2), and its CD spectrum is essentially flat (Figure S6), although a very weak positive couplet $\left(\lambda=450 \mathrm{~nm}, \Delta \varepsilon=1.00 \mathrm{~L}^{\circ} \mathrm{mol}^{-}\right.$ ${ }^{1} \mathrm{~cm}^{-1}$ ) is observed. More significantly, the NMR spectrum of this compound in $\mathrm{D}_{2} \mathrm{O}$ does not show the aggregation-induced large line broadening observed for the Yariv reagents (Supporting Information). Taken together, these three experiments demonstrate that the CD signal of $\mathbf{1}$ in 
aqueous solution arises due to the formation of chiral aggregates rather than due to the inherent chirality of the molecule.

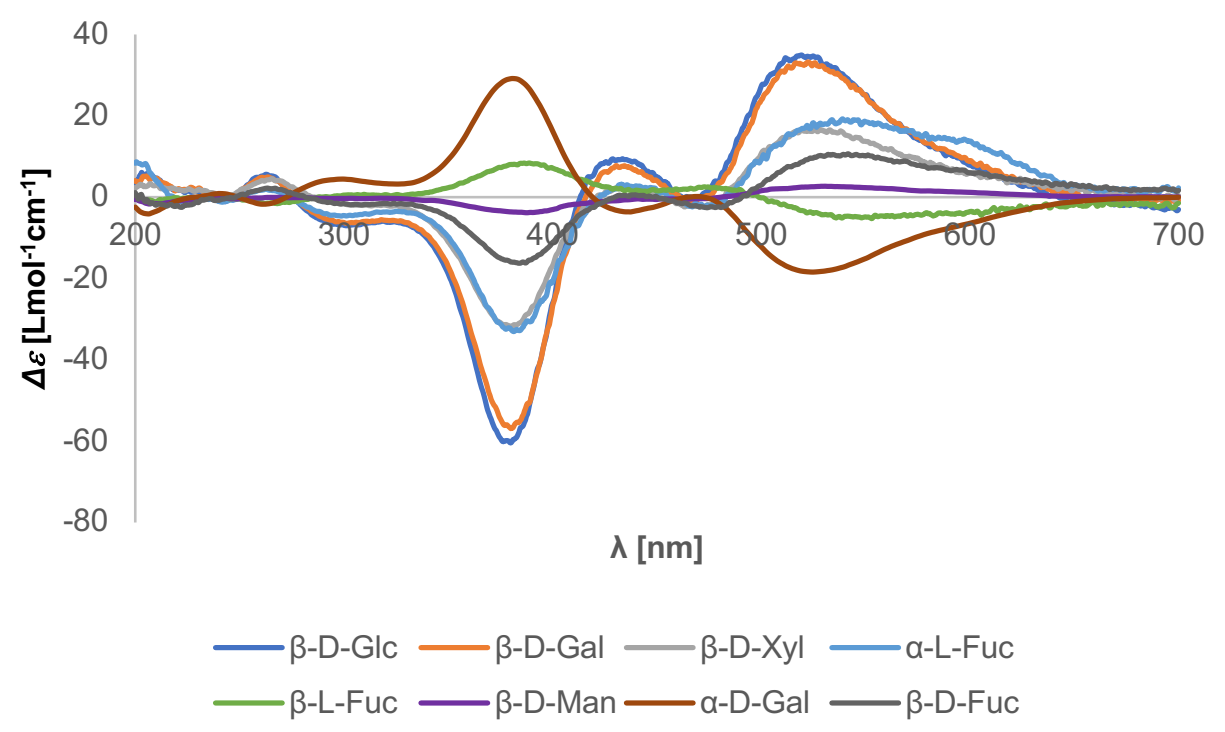

Figure 3. CD spectra of $300 \mu \mathrm{M}$ solutions of various Yariv reagents in water

The CD spectra of compounds $\mathbf{2}-\mathbf{4}$ and $\mathbf{8}$ were obtained in water (Figure 3 ). All the $\beta$-Dsugar variants of the Yariv reagents such as $\beta$-D-galactosyl (2), $\beta$-D-xylosyl (3), $\beta$-D-fucosyl (4) and $\beta$-D-mannosyl (8) derivatives exhibit $\mathrm{CD}$ spectra that are similar in shape to the $\mathrm{CD}$ spectrum of 1. In each case, two overlapping positive CD couplets are observed. The intensities of the various spectra however vary quite dramatically. The intensity of the CD spectra of $2(\lambda=380 \mathrm{~nm}$, $\left.\Delta \varepsilon=-56 \mathrm{~L} \cdot \mathrm{mol}^{-1} \mathrm{~cm}^{-1} ; \lambda=527 \mathrm{~nm}, \Delta \varepsilon=32 \mathrm{~L} \cdot \mathrm{mol}^{-1} \mathrm{~cm}^{-1}\right)$ is quite large and comparable to $\mathbf{1}(\lambda=$ $\left.380 \mathrm{~nm}, \Delta \varepsilon=-57 \mathrm{~L} \mathrm{~mol}^{-1} \mathrm{~cm}^{-1} ; \lambda=527 \mathrm{~nm}, \Delta \varepsilon=34 \mathrm{~L} \cdot \mathrm{mol}^{-1} \mathrm{~cm}^{-1}\right)$. The intensity of the CD spectra of $3\left(\lambda=380 \mathrm{~nm}, \Delta \varepsilon=-32 \mathrm{~L} \cdot \mathrm{mol}^{-1} \mathrm{~cm}^{-1} ; \lambda=527 \mathrm{~nm}, \Delta \varepsilon=17 \mathrm{~L}^{\prime} \mathrm{mol}^{-1} \mathrm{~cm}^{-1}\right)$ and $4(\lambda=380 \mathrm{~nm}, \Delta \varepsilon$ $=-16 \mathrm{~L} \cdot \mathrm{mol}^{-1} \mathrm{~cm}^{-1} ; \lambda=527 \mathrm{~nm}, \Delta \varepsilon=10 \mathrm{~L}^{\prime} \mathrm{mol}^{-1} \mathrm{~cm}^{-1}$ ) is significantly less than $\mathbf{1}$ and $\mathbf{2}$. Finally $\mathbf{8}$ $\left(\lambda=380 \mathrm{~nm}, \Delta \varepsilon=-4 \mathrm{~L} \cdot \mathrm{mol}^{-1} \mathrm{~cm}^{-1} ; \lambda=527 \mathrm{~nm}, \Delta \varepsilon=2.5 \mathrm{~L}^{\prime} \cdot \mathrm{mol}^{-1} \mathrm{~cm}^{-1}\right)$ produces a CD spectrum that is almost negligible in comparison to $\mathbf{1}$ and $\mathbf{2}$. The lower intensity in the $\beta$-D derivative $\mathbf{4}$ may 
arise from its low solubility ${ }^{28}$ in water. However, the almost negligible CD spectrum of $\mathbf{8}$ likely arises either due to the morphology of the aggregates of $\mathbf{8}$ (larger interchromophoric distance or smaller angle between the electric dipole transition moments compared to other Yariv aggregates, i.e. less pronounced right hand helicity) or smaller excess of aggregate helicity in a mixture of both left and right handed aggregates. It has previously been shown that $\mathbf{8}$ does form aggregates ${ }^{9}$ in solution and the UV/vis spectrum of an aqueous solution of $\mathbf{8}$ clearly resembles the spectrum of other Yariv aggregates, which are aggregating conditions (Figure S1), so the low intensity does not arise due to lack of aggregation altogether. Overall, these results suggest that $\beta$-D-sugar containing Yariv reagents form strong helical aggregates with large positive CD couplets $(\lambda=527$ $\mathrm{nm}, \Delta \varepsilon>10.00 \mathrm{~L} \cdot \mathrm{mol}^{-1} \mathrm{~cm}^{-1}$ ) with exception of the $\beta$-D-mannoside. Whether the higher intensities in compounds 1-4 is due to an actual excess of aggregates of one helicity over the other, or due to more pronounced helical CD signatures, awaits further biophysical characterization of the aggregates.

The effect of switching from $\beta$ - anomer to $\alpha$-anomer on the helicity of the Yariv reagent aggregates was tested by obtaining the CD spectra of $\alpha$-D-galacatosyl Yariv 5 , the $\alpha$-anomeric version of 2. Similar to the $\beta$-D sugars containing Yariv (1-4) two bisignate Cotton effects were seen (Figure 3). However, the signs of the Cotton effects were inverted for $5(\lambda=380 \mathrm{~nm}, \Delta \varepsilon=27$ $\left.\mathrm{L} \cdot \mathrm{mol}^{-1} \mathrm{~cm}^{-1} ; \lambda=527 \mathrm{~nm}, \Delta \varepsilon=-17 \mathrm{~L} \cdot \mathrm{mol}^{-1} \mathrm{~cm}^{-1}\right)$ suggesting an inversion of helicity in the aggregates of $\alpha$-D-sugar containing Yarivs compared to $\beta$-D-sugar containing Yarivs. A sample of $\alpha$-D-Glucose Yariv was also prepared but lack of solubility ${ }^{28}$ prevented a CD analysis.

Next, the effect of change from D to L enantiomer was studied by recording the CD spectra for $\beta$-L-fucosyl Yariv (6), the enantiomer of (4). Again, two bi-signate Cotton effects were seen (Figure 3) and the sign of the Cotton effects were reversed compared to $\beta$-D-sugar containing 
Yarivs $\left(\lambda=380 \mathrm{~nm}, \Delta \varepsilon=8 \mathrm{~L} \cdot \mathrm{mol}^{-1} \mathrm{~cm}^{-1} ; \lambda=527 \mathrm{~nm}, \Delta \varepsilon=-5 \mathrm{~L}^{\prime} \mathrm{mol}^{-1} \mathrm{~cm}^{-1}\right)$ indicating a reversal in the helicity of the aggregates of 6 compared to 1-4. The smaller magnitude of the Cotton effect in 6 as well the lack of two peaks in the UV/vis spectra (Figure S1) likely arise from weaker solubility $^{28}$ in water. Finally, the CD spectra of $\alpha$-L-fucosyl Yariv (7) was also recorded. Since 7 is the $\alpha$ anomeric version of $\mathbf{6}$, an opposite CD couplet is expected and this is what was found ( $\lambda$ $=380 \mathrm{~nm}, \Delta \varepsilon=-32 \mathrm{~L} \cdot \mathrm{mol}^{-1} \mathrm{~cm}^{-1} ; \lambda=527 \mathrm{~nm}, \Delta \varepsilon=18 \mathrm{~L} \cdot \mathrm{mol}^{-1} \mathrm{~cm}^{-1}$ ) (Figure 3). These results indicate that change in D or L configuration as well as change in the anomeric stereochemistry causes a reversal in helicity in the Yariv reagent aggregates.

Although it has previously been shown that only certain $\beta$-D-sugar containing Yariv reagents bind $\mathrm{AGP}^{8}$, the $\mathrm{AGP}$ binding ability of these Yariv reagents has never been compared on a single gel. Moreover, AGP binding ability of $\mathbf{4}$ and $\mathbf{6}$ had never been tested previously. The AGP binding abilities of the Yariv reagents 1-7 were tested using the reverse gel assay (A technique we developed that allows easy comparison of AGP binding ability of various Yariv reagents on a single gel, see SI for more information). Only Yariv reagents 1-4 showed formation of concentrated dark red halos upon addition into the reverse gel (Figure S10a). Therefore, as shown previously only $\beta$-D-sugar containing Yariv reagents were found to bind AGP. The $\alpha$-D-galactosyl 5, $\beta$-L-fucosyl 6, and $\alpha$-L-fucosyl 7 Yariv reagents (Figure S10b) and $\beta$-D-mannosyl 8 (tested previously using a regular gel assay) did not display the concentrated dark red halos, i.e. AGP binding ability.

Considering the gel-binding assay results in conjunction with the CD spectra, the ability to bind AGPs correlates strongly with whether a Yariv reagent exhibits a strong positive CD couplet. The two largest halos were formed by the $\beta$-D-glucosyl and $\beta$-D-galactosyl reagents 1 (average diameter $=0.4 \mathrm{~cm})$ and 2 (average diameter $=0.3 \mathrm{~cm})$, which also exhibit the most intense CD 
signals. Analogs $3(\beta$-D-xylosyl, average diameter $=0.26 \mathrm{~cm})$ and $4(\beta$-D-fucosyl, average diameter $=0.23 \mathrm{~cm}$ ) which show a significantly less intense $\mathrm{CD}$ signal than $\mathbf{1}$ and $\mathbf{2}$, also form significantly less pronounced dark red halos in the reverse gel assay. Of particular note is the $\alpha-\mathrm{L}$-fucosyl derivative 7, which does not bind AGP (Figure S10), despite exhibiting a positive CD couplet. This indicates that the formation of aggregates with a large positive CD couplet is not sufficient for eliciting AGP binding. Our results do conclusively show that $\beta$-D-sugar containing Yariv reagents that also show strong positive $\mathrm{CD}$ couplet $\left(\Delta \varepsilon>10 \mathrm{~L} \mathrm{~mol}^{-1} \mathrm{~cm}^{-1}\right.$ at $\left.527 \mathrm{~nm}\right)$ displayed AGP binding ability. Furthermore, the magnitude of AGP binding ability of $\beta$-D-sugar containing Yariv reagents strongly correlates to the magnitude of the Cotton effect in the CD spectra.

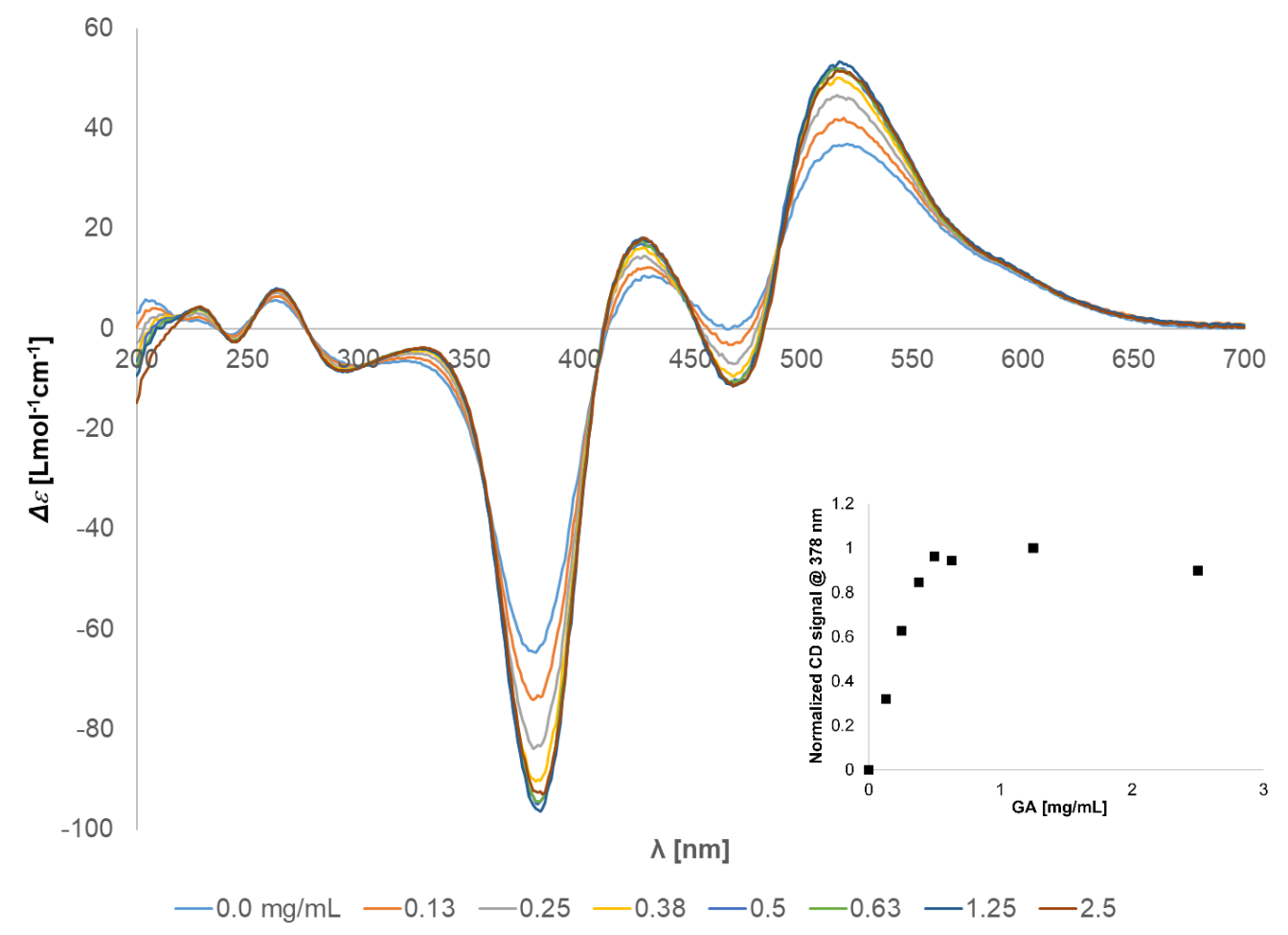

Figure 4. Change in CD spectra of (1) upon addition of GA AGP and inset shows change in ellipticity at $382 \mathrm{~nm}$

Additionally, we also found that the binding of Yariv reagents to AGP can be monitored via $\mathrm{CD}$ and absorbance spectroscopy. The $\mathrm{CD}$ and $\mathrm{UV} /$ vis absorbance spectra of (1) show an 
increase in intensity upon addition of gum arabic (GA) AGP (Figure 4), but not upon addition of frost grape (FG) AGP which is known to not bind ${ }^{29}$ Yariv reagents (Figure S5). Also, there is no change in the spectra of (7) upon addition of GA AGP or FG AGP (Figure S4). The increase in $\mathrm{CD}$ and UV/vis absorbance of (1) levels out at an AGP concentration of $1.25 \mathrm{mg} / \mathrm{mL}$. The exact reason for the increase and subsequent levelling out of the CD signal and UV/vis absorbance is not understood, thus more intensive characterization of the Yariv-AGP complex is required.

In summary, our results are consistent with the proposed hypothesis that the Yariv reagent aggregates are helical. We also demonstrate that strong positive $\mathrm{CD}$ couplet is required for possessing AGP binding ability. Thus, our result which shows the lack of a strong positive CD couplet in $\beta$-D-Mannose Yariv (8), helps explain why unlike other $\beta$-D-sugar containing Yarivs, 8 cannot bind AGP. The Yariv reagents are an example of C3-symmteric molecules, this class of molecules have previously been shown to self-assemble into helical stacks (H aggregates) ${ }^{26,27}$. Further studies will investigate if this is the case for the morphology of the Yariv aggregates as well or whether the Yariv aggregates form higher order aggregates or some other structure. The composition of the aggregates will be explored by confirming the identity of the chromophores in the Yariv UV/vis spectra. More insights into the Yariv-AGP complex are also required.

\section{Acknowledgements}

We gratefully acknowledge support of this research by the National Science Foundation (CHE 1607554). R.L. and S.W. are recipients of Brown University Undergraduate Teaching and Research Awards, and B.W.L. is a Brown University Chemistry Department Dissertation Fellow. We thank Neil Price of the USDA for a sample of FGP. This research is based in part upon work conducted using the Rhode Island NSF/EPSCoR Proteomics Shared Resource Facility, which is 
supported in part by the National Science Foundation (EPSCoR 1004057), National Institutes of Health (1S10RR020923, S10RR027027), a Rhode Island Science and Technology Advisory Council grant, and the Division of Biology and Medicine, Brown University. Students in Advanced Organic Chemistry (CHEM 1450, Spring 2015) are thanked for their assistance with developing protocols for the synthesis of $\mathbf{S 2}$.

\section{References}

1. Classen, B.; Baumann, A.; Utermoehlen, J., Arabinogalactan-proteins in spore-producing land plants. Carbohydr Polym 2019, 210, 215-224.

2. $\quad$ Pereira, A. M.; Pereira, L. G.; Coimbra, S., Arabinogalactan proteins: rising attention from plant biologists. Plant Reprod 2015, 28 (1), 1-15.

3. Seifert, G. J.; Roberts, K., The biology of arabinogalactan proteins. Annu Rev Plant Biol 2007, 58, 137-61.

4. Lopez, R. A.; Renzaglia, K. S., Arabinogalactan proteins and arabinan pectins abound in the specialized matrices surrounding female gametes of the fern Ceratopteris richardii. Planta 2016, 243 (4), 947-57.

5. Motose, H.; Sugiyama, M.; Fukuda, H., A proteoglycan mediates inductive interaction during plant vascular development. Nature 2004, 429 (6994), 873-8.

6. Kitazawa, K.; Tryfona, T.; Yoshimi, Y.; Hayashi, Y.; Kawauchi, S.; Antonov, L.;

Tanaka, H.; Takahashi, T.; Kaneko, S.; Dupree, P.; Tsumuraya, Y.; Kotake, T., beta-galactosyl

Yariv reagent binds to the beta-1,3-galactan of arabinogalactan proteins. Plant Physiol 2013, 161 (3), 1117-26.

7. Paulsen, B. S.; Craik, D. J.; Dunstan, D. E.; Stone, B. A.; Bacic, A., The Yariv reagent: behaviour in different solvents and interaction with a gum arabic arabinogalactan-protein. Carbohydr Polym 2014, 106, 460-8.

8. Jermyn, Comparative specificity of concanavalin A and the beta lectins. Aust. J. Plant Physiol. 1978, 687-696.

9. $\quad$ E.F. Woods, G. G. L., M.A. Jermyn, The Self-Association of Glycosyl Phenylazo Dyes (Yariv Antigens). Aust. J. Chem. 1978, 31 (10), 2225-2238.

10. Zhou, L. H.; Weizbauer, R. A.; Singamaneni, S.; Xu, F.; Genin, G. M.; Pickard, B. G., Structures formed by a cell membrane-associated arabinogalactan-protein on graphite or mica alone and with Yariv phenylglycosides. Ann Bot 2014, 114 (6), 1385-97.

11. Hendrikse, S. I. S.; Su, L.; Hogervorst, T. P.; Lafleur, R. P. M.; Lou, X.; van der Marel, G. A.; Codee, J. D. C.; Meijer, E. W., Elucidating the Ordering in Self-Assembled Glycocalyx Mimicking Supramolecular Copolymers in Water. J Am Chem Soc 2019, 141 (35), 13877-13886. 12. Hu, J.; Kuang, W.; Deng, K.; Zou, W.; Huang, Y.; Wei, Z.; Faul, C. F. J., SelfAssembled Sugar-Substituted Perylene Diimide Nanostructures with Homochirality and High Gas Sensitivity. Adv. Funct. Mater. 2012, 22 (19), 4149-4158.

13. Huang, Y.; Hu, J.; Kuang, W.; Wei, Z.; Faul, C. F., Modulating helicity through amphiphilicity-tuning supramolecular interactions for the controlled assembly of perylenes. Chem Commun (Camb) 2011, 47 (19), 5554-6. 
14. Huang, Y.; Wang, J.; Wei, Z., Modulating supramolecular helicity and electrical conductivity of perylene dyes through an achiral alkyl chain. Chem Commun (Camb) 2014, 50 (61), 8343-5.

15. Liu, X.; Huang, Z.; Huang, Y.; Zhu, L.; Fu, J., Tunable Supramolecular Helical Aggregate and Optoelectrical Properties of Perylene Diimides by Stereoisomerism of Sugar. $J$. Phys. Chem. C 2017, 121 (13), 7558-7563.

16. Sun, K.; Xiao, C.; Liu, C.; Fu, W.; Wang, Z.; Li, Z., Thermally sensitive self-assembly of glucose-functionalized tetrachloro-perylene bisimides: from twisted ribbons to microplates. Langmuir 2014, 30 (37), 11040-5.

17. Wang, K. R.; An, H. W.; Wang, Y. Q.; Zhang, J. C.; Li, X. L., Multivalent glycoclusters constructed by chiral self-assembly of mannose functionalized perylene bisimide. Org Biomol Chem 2013, 11 (6), 1007-12.

18. Wang, K. R.; An, H. W.; Wu, L.; Zhang, J. C.; Li, X. L., Chiral self-assembly of lactose functionalized perylene bisimides as multivalent glycoclusters. Chem Commun (Camb) 2012, 48 (45), 5644-6.

19. Wang, K. R.; Han, D.; Cao, G. J.; Li, X. L., Synthesis and predetermined supramolecular chirality of carbohydrate-functionalized perylene bisimide derivatives. Chem Asian J 2015, 10 (5), 1204-14.

20. Wang, K.-R.; Han, D.; Cao, G.-J.; Li, X.-L., Link spacer controlled supramolecular chirality of perylene bisimide-carbohydrate conjugate. RSC Advances 2015, 5 (59), 4772847731.

21. Hoshing, R.; Saladino, M.; Kuhn, H.; Caianiello, D.; Lusi, R. F.; Basu, A., An Improved Protocol for the Synthesis and Purification of Yariv Reagents. J Org Chem 2020.

22. Lee, H. Y.; Song, X.; Park, H.; Baik, M. H.; Lee, D., Torsionally responsive C3symmetric azo dyes: azo-hydrazone tautomerism, conformational switching, and application for chemical sensing. J Am Chem Soc 2010, 132 (34), 12133-44.

23. Chen, X. C.; Tao, T.; Wang, Y. G.; Peng, Y. X.; Huang, W.; Qian, H. F., Azo-hydrazone tautomerism observed from UV-vis spectra by $\mathrm{pH}$ control and metal-ion complexation for two heterocyclic disperse yellow dyes. Dalton Trans 2012, 41 (36), 11107-15.

24. Hestand, N. J.; Spano, F. C., Expanded Theory of H- and J-Molecular Aggregates: The Effects of Vibronic Coupling and Intermolecular Charge Transfer. Chem Rev 2018, 118 (15), 7069-7163.

25. Swathi, K.; Sissa, C.; Painelli, A.; George Thomas, K., Supramolecular chirality: a caveat in assigning the handedness of chiral aggregates. Chem Commun (Camb) 2020, 56 (59), 82818284.

26. Buendia, J.; Calbo, J.; Garcia, F.; Arago, J.; Viruela, P. M.; Orti, E.; Sanchez, L., Helical supramolecular polymerization of $\mathrm{C} 3$-symmetric amides and retroamides: on the origin of cooperativity and handedness. Chem Commun (Camb) 2016, 52 (42), 6907-10.

27. Van Gorp, J. J.; Vekemans, J. A.; Meijer, E. W., C3-symmetrical supramolecular architectures: fibers and organic gels from discotic trisamides and trisureas. J Am Chem Soc 2002, 124 (49), 14759-69.

28. Jermyn, M.; Yeow, Y.; Woods, E., A Class of Lectins Present in the Tissues of Seed Plants. Funct. Plant Biol. 1975, 2 (4), 501-531.

29. Price, N. P.; Vermillion, K. E.; Eller, F. J.; Vaughn, S. F., Frost Grape Polysaccharide (FGP), an Emulsion-Forming Arabinogalactan Gum from the Stems of Native North American Grape Species Vitis riparia Michx. J Agric Food Chem 2015, 63 (32), 7286-93. 


\section{Supporting Information}

\section{The chirality of Yariv reagent aggregates correlates with AGP-binding ability}

Raghuraj Hoshing, Blaise W Leeber III, Helene Kuhn, David Caianiello, Brandon Dale, Michael Saladino, Robert Lusi, Natalie Palaychuk, Sarah Weingarten, and Amit Basu*

Department of Chemistry, Brown University, Providence RI, USA 02912

abasu@brown.edu

\section{General Experimental Protocols}

Thin layer chromatography was carried out on Merck silica gel $60 \mathrm{~F}_{254}$ precoated glass plates. Spots were visualized under a UV lamp and/or by immersion in $p$-anisaldehyde stain. All NMR spectra were recorded on Bruker Avance III HD Ascend $600 \mathrm{MHz} .{ }^{1} \mathrm{H}-\mathrm{NMR}$ and ${ }^{13} \mathrm{C}-\mathrm{NMR}$ spectra were referenced to residual solvent peaks. Coupling constants are given in [Hz] and chemical shifts in [ppm]. Electrospray ionization (ESI) mass spectra were obtained using a Thermo LCQ Deca XP Max ion trap mass spectrometer. Purified water was obtained from a EMD Millipore Direct- $\mathrm{Q}^{\mathrm{TM}}$ 3 Tap to Pure and Ultrapure Water Purification System. Acacia gum arabic was purchased from Sigma-Aldrich. Frost grape polysaccharide (FGP) was generously provided by Dr. Neil Price (USDA, Peoria). 
<smiles>Nc1ccc(O[C@@H]2O[C@H](CO)[C@@H](O)[C@H](O)[C@H]2O)cc1</smiles>

$\alpha$-D-galactosyl-Yariv (5)

p-aminophenyl- $\alpha$-D-galactopyranoside $(218 \mathrm{mg}, 0.81 \mathrm{mmol})$ was dissolved in $0.5 \mathrm{M} \mathrm{HCl}(4.7 \mathrm{~mL})$ and cooled to $0{ }^{\circ} \mathrm{C}$. A freshly prepared cold aqueous solution of $0.3 \mathrm{M} \mathrm{NaNO}_{2}(2.7 \mathrm{~mL})$ was added with an addition funnel over a period of $5 \mathrm{~min}$, and the solution was stirred for $2 \mathrm{~h}$. The reaction mixture was cooled to $0{ }^{\circ} \mathrm{C}$ and a solution of phloroglucinol $(31 \mathrm{mg}, 0.25 \mathrm{mmol}, 0.3 \mathrm{eq}$.) in cold water $(10 \mathrm{~mL})$, was added dropwise with an addition funnel over a period of $15 \mathrm{~min}$. The $\mathrm{pH}$ of the reaction mixture was adjusted to 9 with $0.5 \mathrm{M} \mathrm{NaOH}$. The reaction mixture was stirred for an additional $2 \mathrm{~h}$, while readjusting the $\mathrm{pH}$ to 9 if necessary. After $2 \mathrm{~h} \mathrm{MeOH}(25 \mathrm{~mL})$ was added to the reaction mixture and the reaction mixture was placed in a freezer overnight. The $\mathrm{pH}$ was adjusted to neutral with $1 \mathrm{M} \mathrm{HCl}$ and the reaction mixture was placed in a freezer overnight. The formed precipitate was collected using a Hirsch-funnel and rinsed with cold methanol, and dried under high vacuum overnight to provide 7 as a deep red powder (186 mg 0.19 mmol, $76 \%$ yield).

${ }^{1} \mathrm{H}$ NMR $\left(600 \mathrm{MHz}, \mathrm{DMSO}-d_{6}\right) \delta=15.93(\mathrm{~s}, 3 \mathrm{H}), 7.64(\mathrm{~d}, J=8.9,6 \mathrm{H}), 7.22(\mathrm{~d}, J=8.5,6 \mathrm{H}),$, $(\mathrm{d}, J=3.2,3 \mathrm{H}), 4.94(\mathrm{~d}, J=6.0,3 \mathrm{H}), 4.77(\mathrm{~d}, J=5.2,3 \mathrm{H}), 4.58(\mathrm{~d}, J=3.4,3 \mathrm{H}), 4.55$ (t, $J=5.9,3 \mathrm{H})$, $3.79(\mathrm{~m}, 9 \mathrm{H}), 3.72(\mathrm{t}, J=6.3,3 \mathrm{H}), 3.50(\mathrm{~m}, 3 \mathrm{H}), 3.42(\mathrm{~m}, 3 \mathrm{H})$.

${ }^{13} \mathrm{C}$ NMR (151 MHz, DMSO-d $) \delta$ 178.0, 156.8, 136.1, 128.5 118.9, 118.6, 98.8, 72.8, 69.8, 69.1, $68.4,60.9$.

HRMS Calc. for $\mathrm{C}_{42} \mathrm{H}_{49} \mathrm{~N}_{6} \mathrm{O}_{21}[\mathrm{M}+\mathrm{H}]:$ 973.2951, Obsd 973.2925 


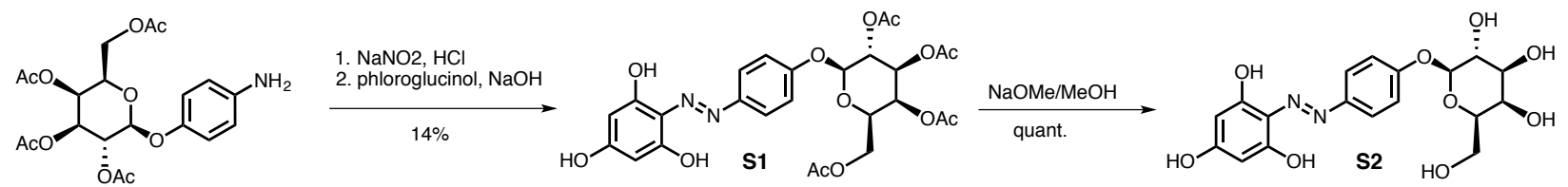

2,3,4,6-Tetraacetyl- $p$-aminophenyl- $\beta$-D-galactopyranoside (1.52 g, $3.5 \mathrm{mmol})$ was dissolved in acetic acid $(20.8 \mathrm{ml})$. and cooled to $0{ }^{\circ} \mathrm{C}$. Hydrochloric acid $(0.89 \mathrm{ml})$ was added, followed by a freshly prepared cold aqueous solution of $0.3 \mathrm{M} \mathrm{NaNO}_{2}(18 \mathrm{~mL})$ was added, and the solution was stirred for $70 \mathrm{~min}$. A cold aqueous solution of phloroglucinol $(4.54 \mathrm{~g}, 36 \mathrm{mmol}, 10 \mathrm{eq})$ was prepared in approximately $350 \mathrm{~mL}$ water. The diazo compound was then rapidly added to the phloroglucinol, with vigorous stirring. Stirring was continued for $10 \mathrm{~min}$, at which point potassium iodide $(0.90 \mathrm{~g}, 5.4 \mathrm{mmol})$ was added, and the reaction was stirred for an additional 10 minutes. The reaction mixture was diluted with dichloromethane and washed with water. The aqueous layer was re-extracted with dichloromethane. The combined organic layers were washed 4 times with water, once with saturated sodium bicarbonate, and once with brine. The organic layer was dried (sodium sulfate) and concentrated in vacuo. The product was obtained after silica gel chromatography (7:3 ethyl acetate:hexane) to provide $\mathbf{S 1}$ as a yellow solid (274 $\mathrm{mg}, .48 \mathrm{mmol}, 14 \%$ )

${ }^{1} \mathrm{H}$ NMR (600MHz, $\left.\mathrm{CDCl}_{3}\right) \delta 13.75$ (br s), $7.60(\mathrm{~m}, 2 \mathrm{H}), 7.02(\mathrm{~m}, 2 \mathrm{H}), 5.99$ (br s, 2H), $5.48(\mathrm{~m}$, $2 \mathrm{H}), 5.15(\mathrm{~m}, 2 \mathrm{H}), 4.23(\mathrm{~m} 1 \mathrm{H}), 4.10(\mathrm{~m}, 1 \mathrm{H}), 2.18,2.08,2.05,2.02(\mathrm{~s}, 12 \mathrm{H})$

${ }^{13} \mathrm{C}$ NMR $\left(125 \mathrm{MHz}, \mathrm{CDCl}_{3}\right) \delta 170.9,170.6,169.9,164.8,158.1,157.8,145.0,122.5,122.3$, $117.4,99.0,96.1,94.5,71.1,70.9,68.7,67.0,61.5,20.8,20.71,20.65,20.63$.

S1 $(15 \mathrm{mg}, 0.026 \mathrm{mmol})$ was dissolved in methanol $(0.15 \mathrm{~mL})$. Freshly prepared $1 \mathrm{M} \mathrm{NaOMe}$ was added $(2.0 \mu \mathrm{L})$ and the solution was stirred at room temperature. The reaction mixture was monitored by TLC (5:1 DCM/methanol). After $6 \mathrm{~h}$ no conversion was seen, $4 \mu \mathrm{NaOMe}$ and 0.15 $\mathrm{mL}$ methanol were added. At $22 \mathrm{~h}$ there was still no conversion, so $\mathrm{NaOMe}$ was added until the $\mathrm{pH}$ of the solution was 10 . The solution was stirred for 2 more hours, at which TLC showed full conversion. The solution was diluted with methanol $(10 \mathrm{~mL})$ and neutralized with Amberlite IR120 ion exchange resin. The mixture was filtered and washed with methanol. Concentration in vacuo provided $\mathbf{S} 2$ quantitatively $(11 \mathrm{mg}, 0.026 \mathrm{mmol})$

${ }^{1} \mathrm{H}$ NMR $\left(600 \mathrm{MHz}, \mathrm{CDCl}_{3}\right) \delta 12.0(\mathrm{br} \mathrm{s}, 3 \mathrm{H}), 7.91(\mathrm{~d}, J=10.2,2 \mathrm{H}), 7.14(\mathrm{~d}, J=8.9,2 \mathrm{H}), 5.86(\mathrm{~s}$, $2 \mathrm{H}), 5.22(\mathrm{~d}, J=4.9,1 \mathrm{H}), 4.93(\mathrm{~d}, J=7.1,1 \mathrm{H}), 4.91(\mathrm{~d}, J=5.2,1 \mathrm{H}), 4.69(\mathrm{t}, J=4.8,1 \mathrm{H}), 4.54$ $(\mathrm{d}, J=4.1,1 \mathrm{H}), 3.71(\mathrm{~m}, 1 \mathrm{H}), 3.65-3.42(\mathrm{~m}, 5 \mathrm{H})$

HRMS Calc. for $\mathrm{C}_{18} \mathrm{H}_{21} \mathrm{~N}_{2} \mathrm{O}_{9}[\mathrm{M}+\mathrm{H}]$ : 409.1247, Obsd. 409.1246

\section{Circular Dichroism/ UV-vis Spectroscopy}

Circular dichroism (CD) and UV/vis measurements were performed in triplicate using a Jasco J815 spectropolarimeter. Temperature was controlled by a JASCO Peltier temperature control unit. Unless otherwise noted the concentration and volume of the Yariv reagent samples was $300 \mu \mathrm{M}$ and $600 \mu \mathrm{L}$, respectively. The sample cell was kept at $20{ }^{\circ} \mathrm{C}$. $\mathrm{CD} / \mathrm{UV}$-vis spectroscopy measurements were obtained in a Hellma analytics $2 \mathrm{~mm}$ pathlength stoppered cuvette and corrected against a purified water standard. Wavelength readings ranged from 200 to $700 \mathrm{nM}$ and were obtained at a speed of $100 \mathrm{nM} /$ minute. The measured ellipticity $\theta$ was converted to molar 
circular dichroism $\Delta \varepsilon=\theta / 32980^{\bullet} c \bullet$, where ellipticity is given in mdeg, $c$ is the concentration in $\mathrm{mol} \cdot \mathrm{L}^{-1}$ and $l$ is the optical path length in $\mathrm{cm}$.

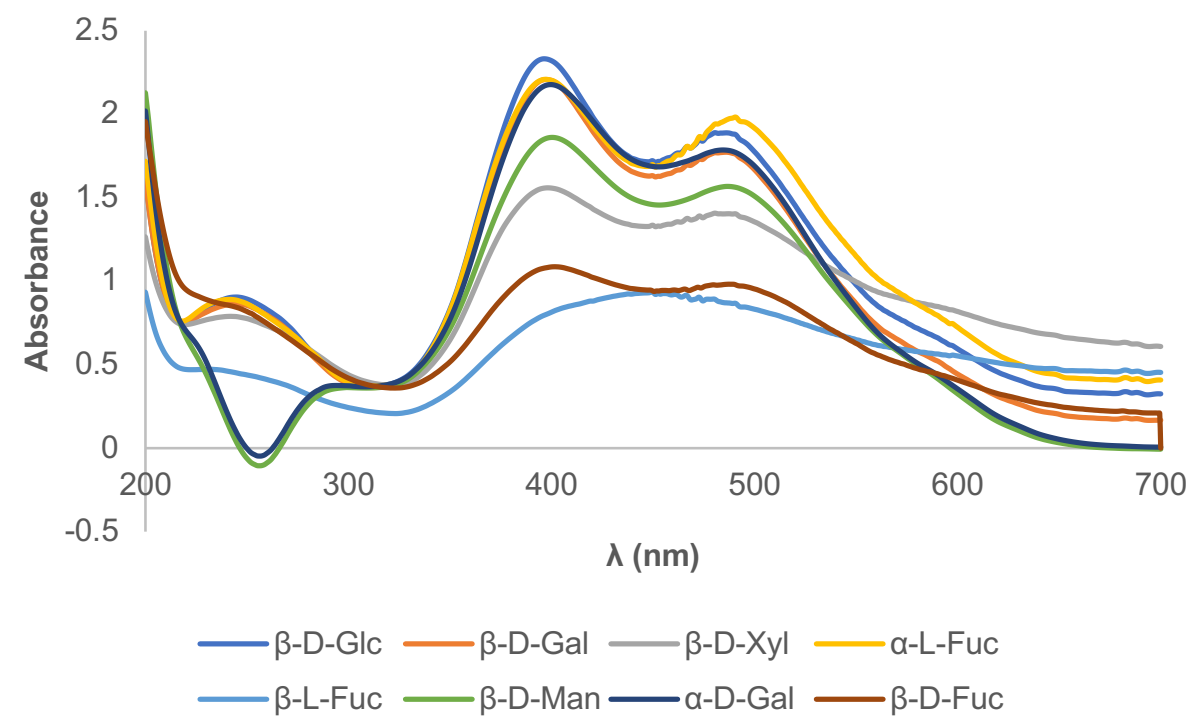

Figure S1. Absorbance spectrum of (1)-(8) (300 $\mu \mathrm{M})$

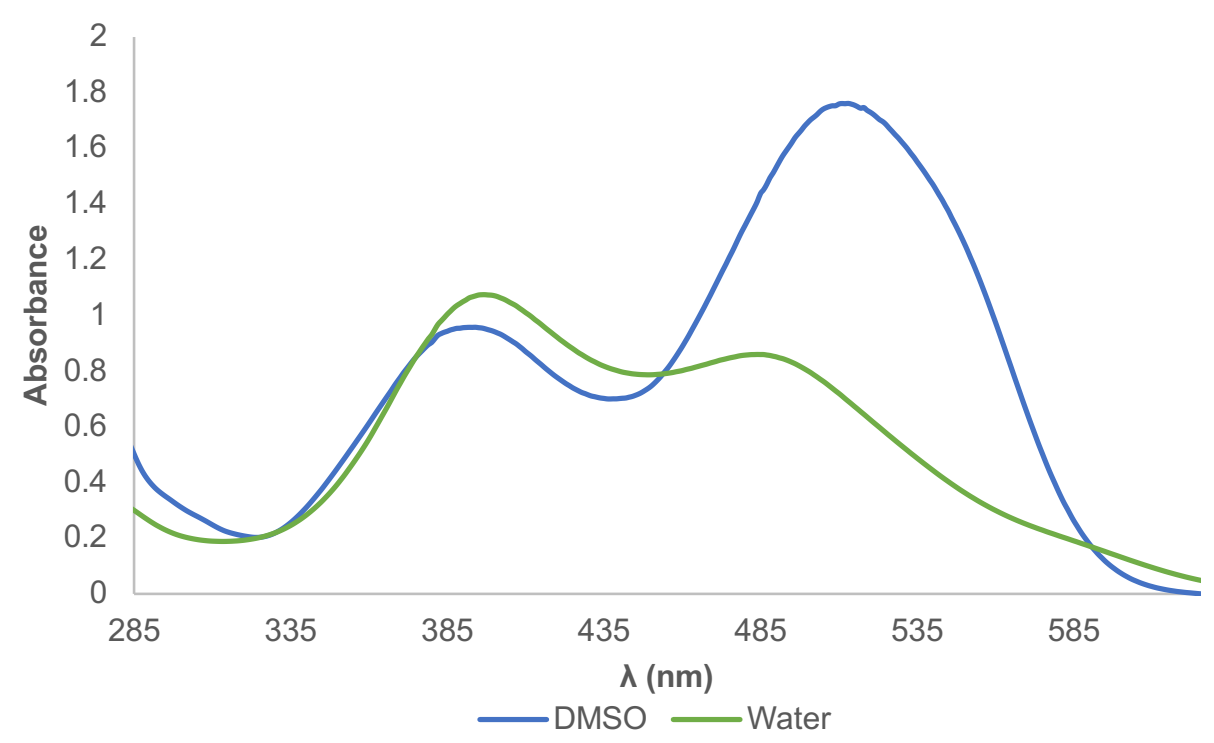

Figure S2. Absorbance spectrum of (1) in DMSO and water $(300 \mu \mathrm{M})$ 


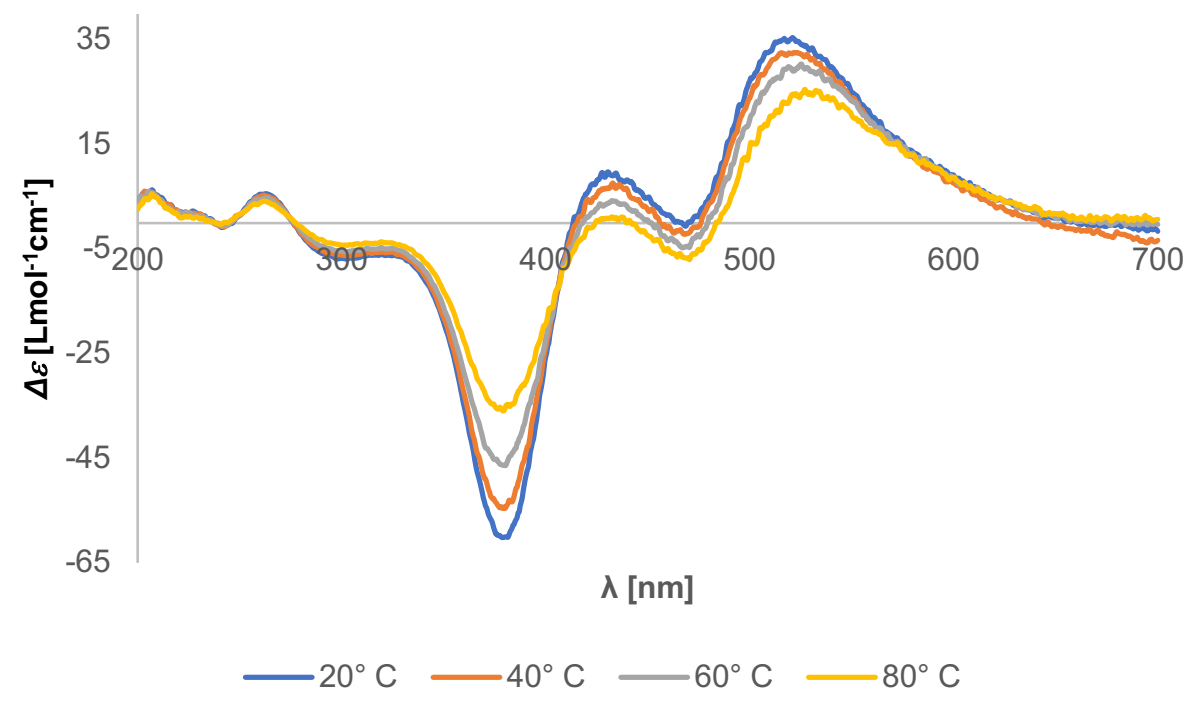

Figure S3. CD spectrum of (1) in water $(300 \mu \mathrm{M})$ at various temperatures

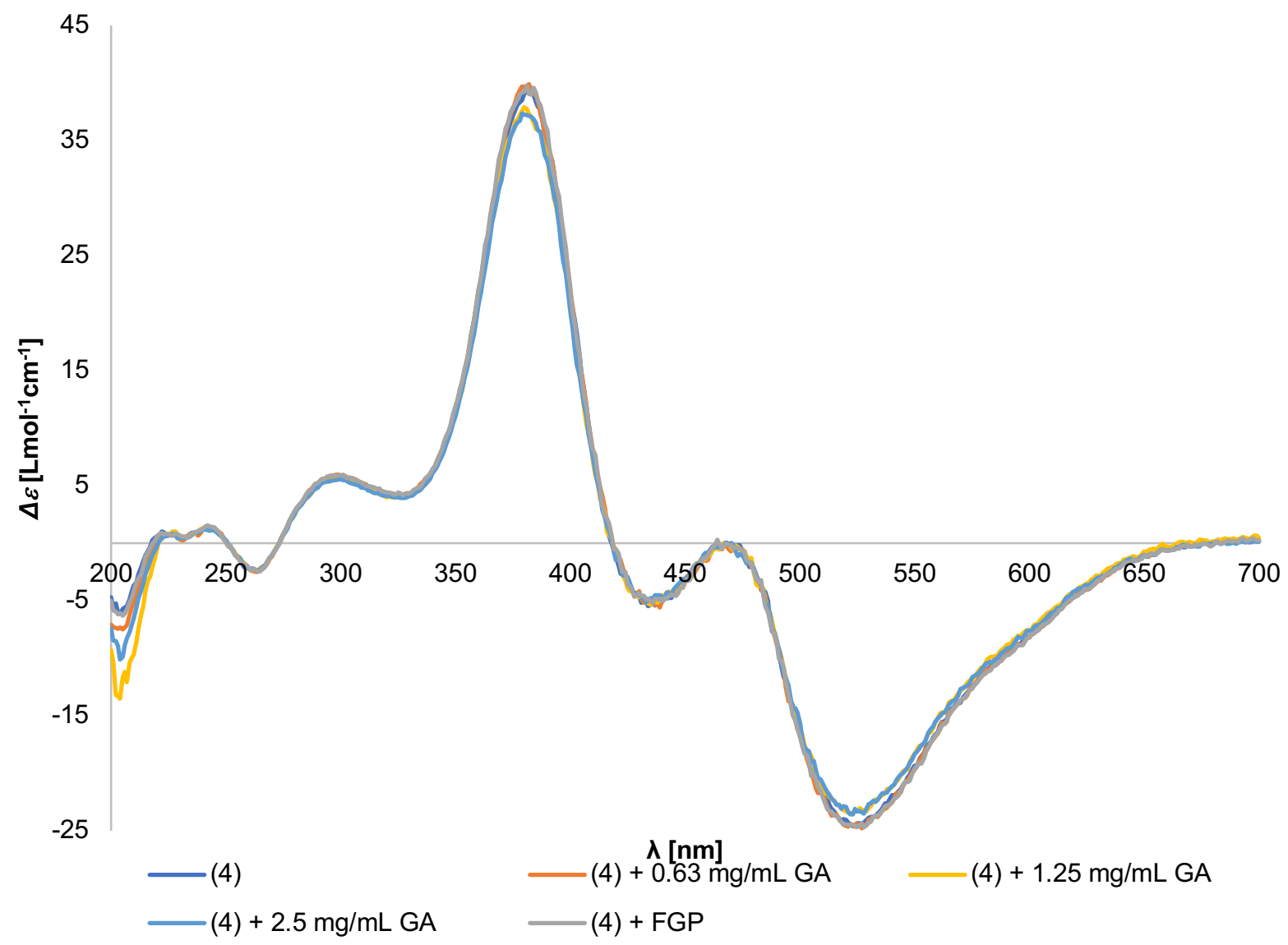

Figure S4 - CD spectra of $5(300 \mu \mathrm{M})$ in the presence of GA $(0.63,1.25$, or $2.50 \mathrm{mg} / \mathrm{mL})$ or FGP $(0.63 \mathrm{mg} / \mathrm{mL})$ 


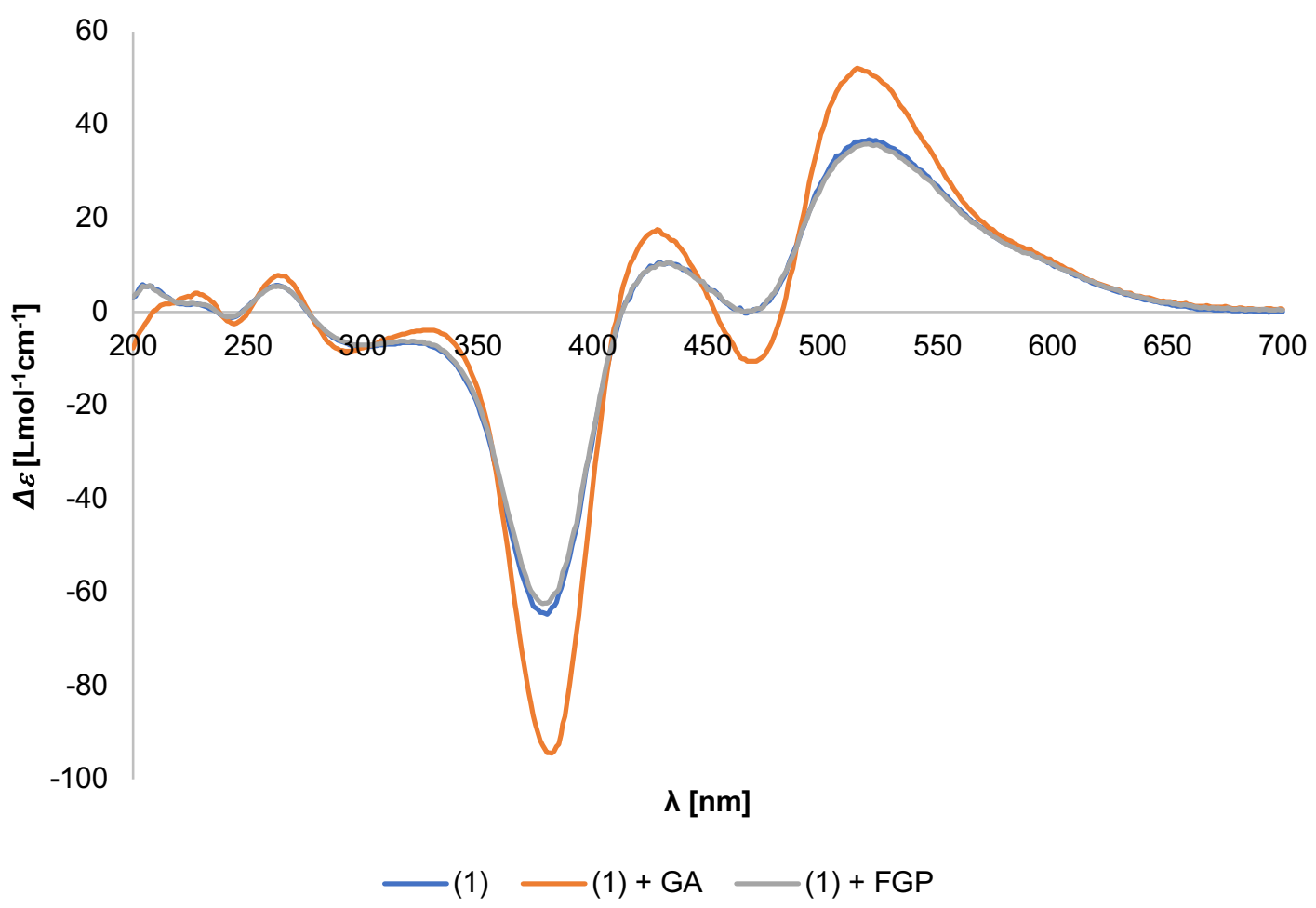

Figure S5 - CD spectra of $1(300 \mu \mathrm{M})$ in the presence of GA $(0.63 \mathrm{mg} / \mathrm{mL})$ or FGP $(0.63$ $\mathrm{mg} / \mathrm{mL}$ )

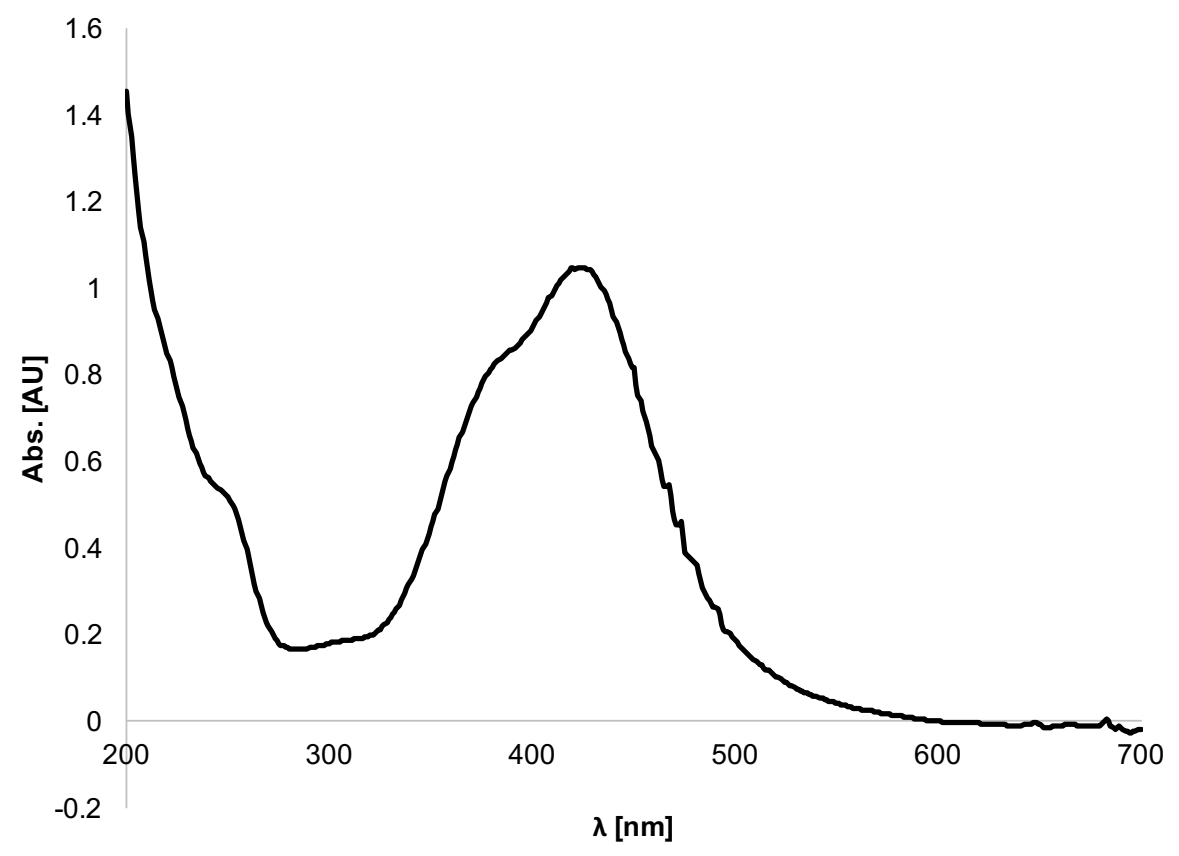




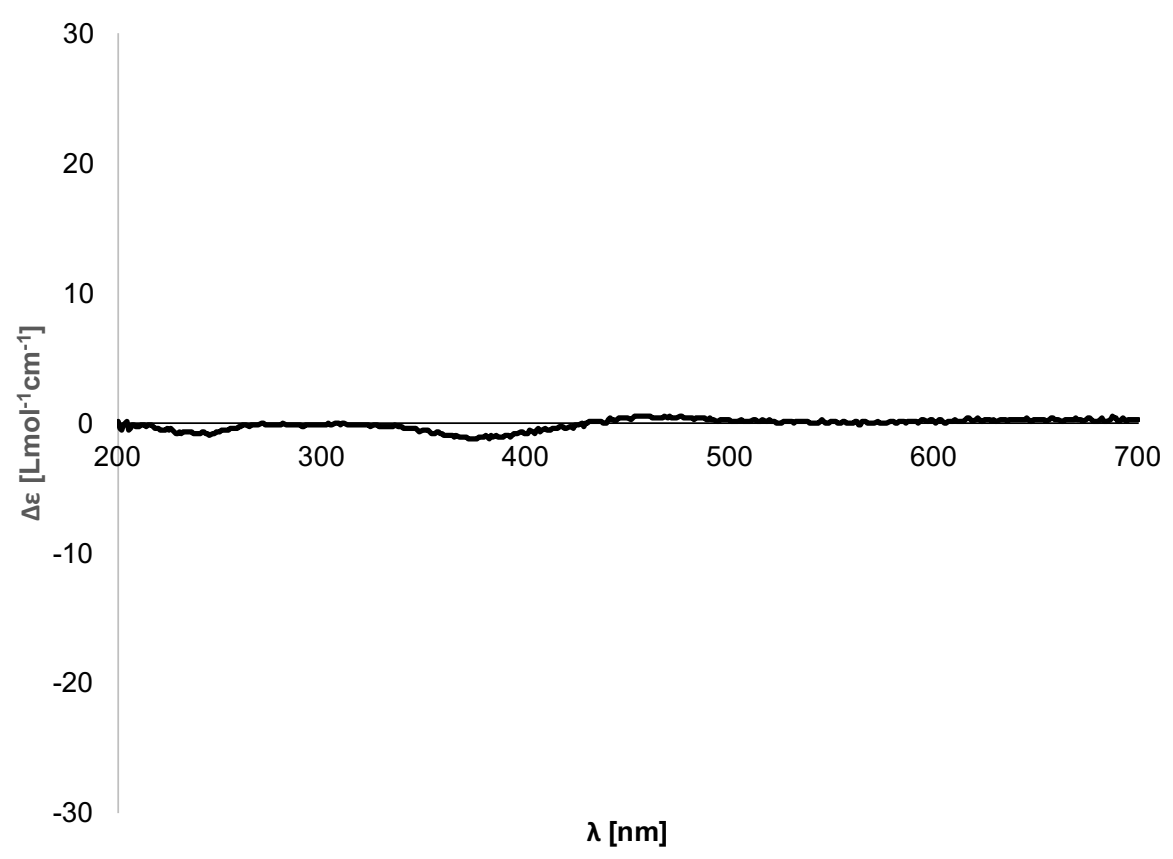

Figure S6 - top) Absorbance spectrum of S2 $(300 \mu \mathrm{M})$; bottom) CD spectrum of S2 $(300 \mu \mathrm{M})$

\section{Gel Assay Protocol:}

In general, $10 \mathrm{~mL}$ of $0.15 \mathrm{M} \mathrm{NaCl}$ was added to $0.10 \mathrm{~g}$ agarose and then $0.1 \mathrm{ml}$ of $1.04 \mathrm{mM} \beta$ glucosyl Yariv reagent was added to the mixture. The solution was heated until boiling $(\sim 30$ secs) in the microwave. Immediately after, $0.1 \mathrm{ml} 0.02 \% \mathrm{w} / \mathrm{v} \mathrm{NaN} \mathrm{N}_{3}$ was added to the solution and mixed slightly. The solution was quickly dispersed into a glass petri dish. $4 \mathrm{ml}$ of the solution was added to the petri dish using a pipet. Once the solution was added to each dish, a custom-made well mold device was inserted into the dish to create the wells in which gum arabic (GA) solution could be added into the gel. The well molds were removed after the gels set for 10 minutes, leaving a gel with 13 wells that could be used to house GA. GA was the inserted into each well using a $10 \mu \mathrm{L}$ Hamilton Microliter ${ }^{\mathrm{TM}}$ PGC syringe; $1 \mu 1$ of GA solution was added into each well. The gels were placed on a platform in a large basin filled with water and covered with foil, to create a moist environment that would prevent the gels from drying out overnight. The gels were left for approximately 16 hours before imaging the next day.

Note: $0.1 \mathrm{~g}$ SDS was added to the gel mixture before heating in the $1 \%$ SDS gel. 


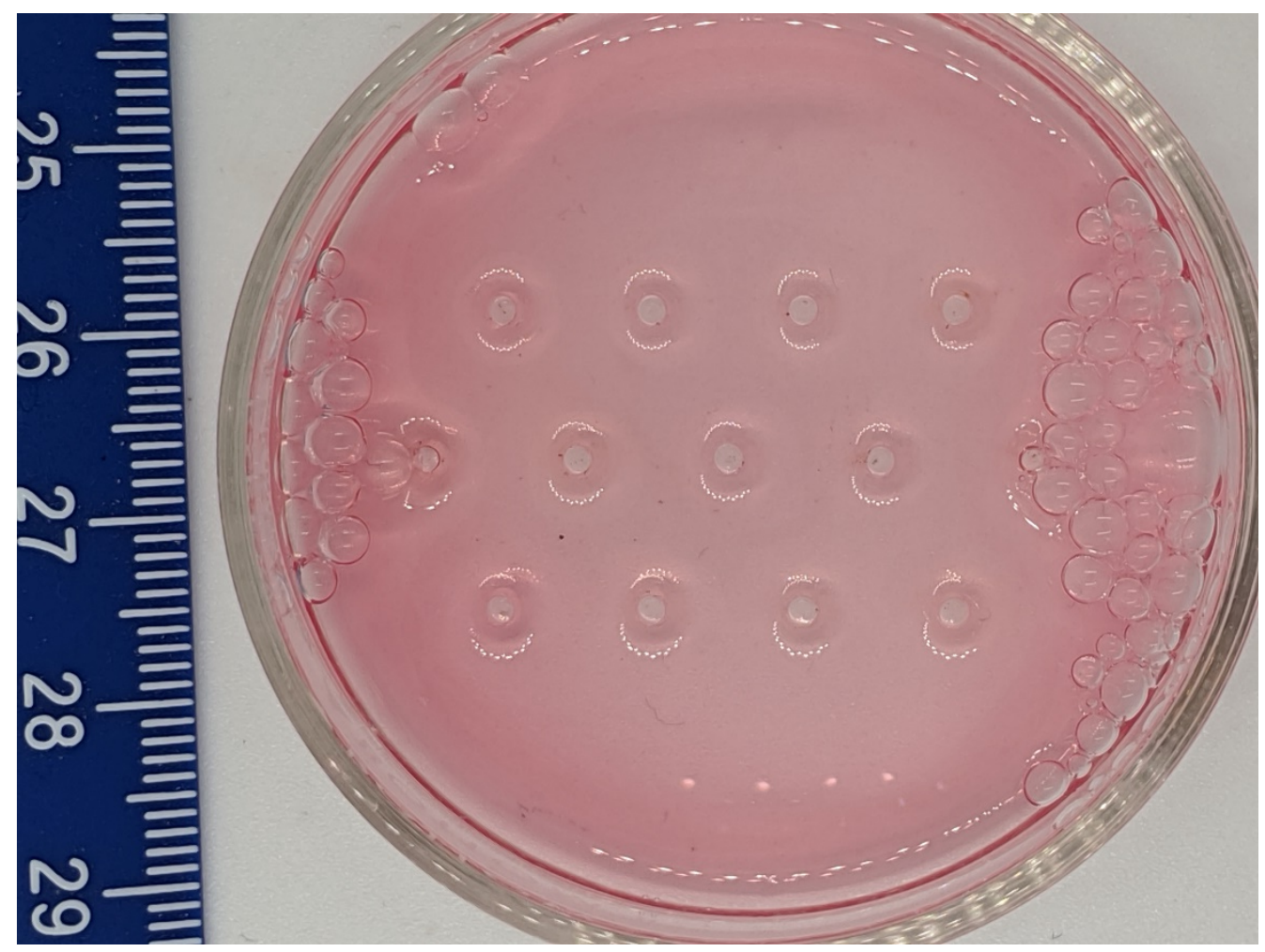

Figure S7 - Gel assay containing 1\%SDS

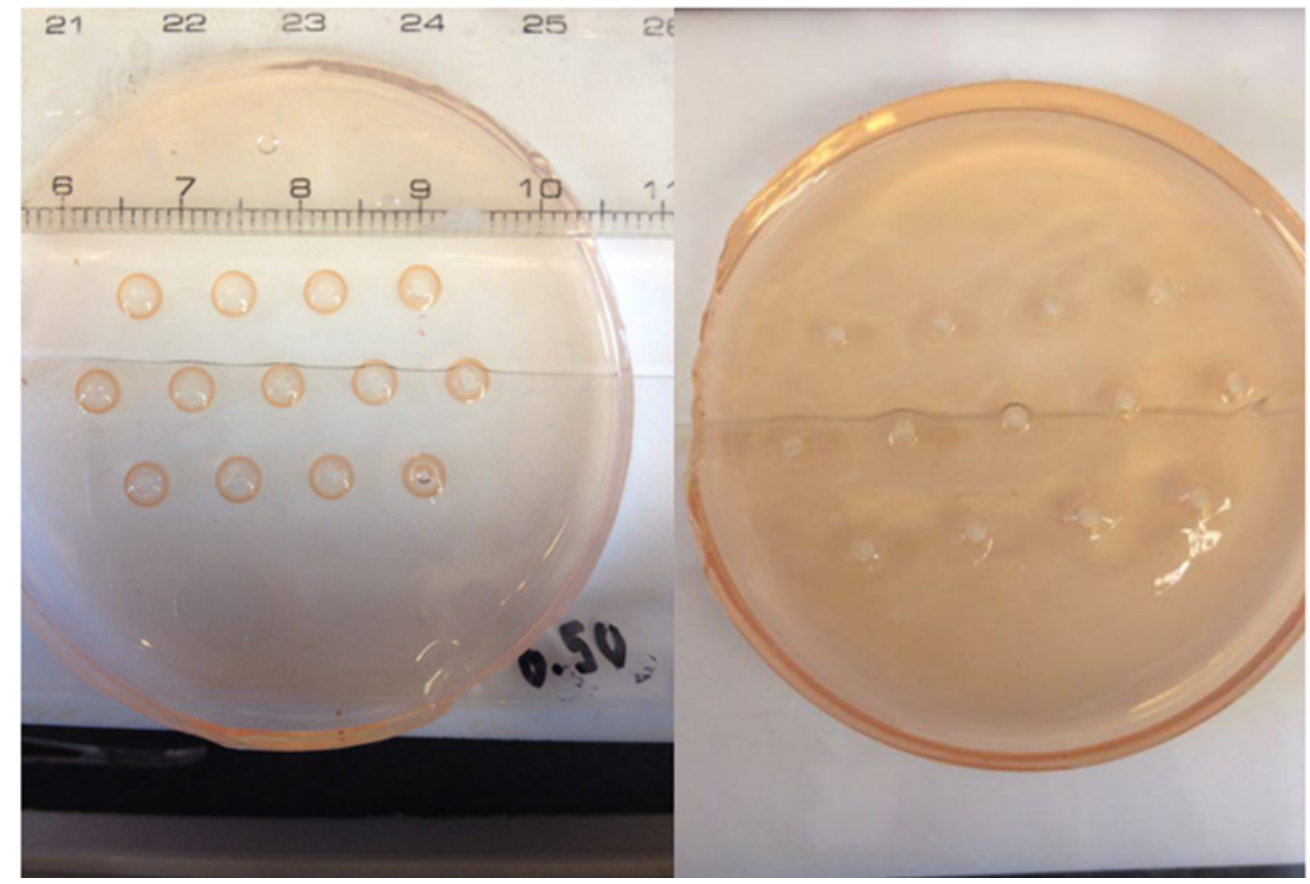

Figure S8. Radial Gel Diffusion Assays with agarose gels containing 1 - left) agarose gels injected with $0.50 \mathrm{~g} / \mathrm{L}$ gum arabic: clear halos are visible at the end of the incubation period; right) agarose gels injected with $1 \mathrm{~g} / \mathrm{L}$ frost grape polysaccharide: no halos are visible. 


\section{Reverse Radial Gel Diffusion Assay}

AGP binding ability has historically been assessed using a gel diffusion assay. The gel diffusion assay was a method introduced for quantification of AGP amount in plant extracts. The method involves addition of AGP solutions of various concentrations into wells in an agarose gel containing Yariv reagent. Red halos form around the wells if AGP binding ability is present, the diameter of the halos is directly correlated to the concentration of AGP solution added to the well. However, comparison of AGP binding ability of different Yariv reagents is not easily carried out using the standard gel assay, as it requires making a unique gel for each Yariv, which renders makes comparisons across gels comprised of different Yariv reagent problematic. We therefore developed a 'reverse' gel assay in which the gel is infused with gum arabic AGP, and the Yariv reagents are added to the wells (Figure S9). This method allows direct comparison of AGP binding ability and also utilizes significantly lower amount of Yariv reagent ( $\sim 10$ times). Since the Yariv reagent diffuses through the gel until all its AGP binding ability is saturated, a larger halo is formed with larger AGP binding ability. The AGP binding ability of different Yariv reagents can thus be compared on single gel because the size of the halo formed directly correlates to AGP binding ability. When no AGP binding ability is present however, the Yariv reagent stays dissolved in the gel and forms a diffuse orange halo rather than precipitate and form a concentrated dark red halo.

The reverse gel assay was optimized by testing various gum arabic concentrations in the gel along with a gradient of various concentrations of $\beta$-D-Glucose Yariv in the wells. It was seen that when using gum arabic concentrations of $0.05 \mathrm{~g} / \mathrm{L}$ gum arabic and $1.04 \mathrm{mM}$ Yariv the sizes of the halos produced were comparable to regular gel assays.

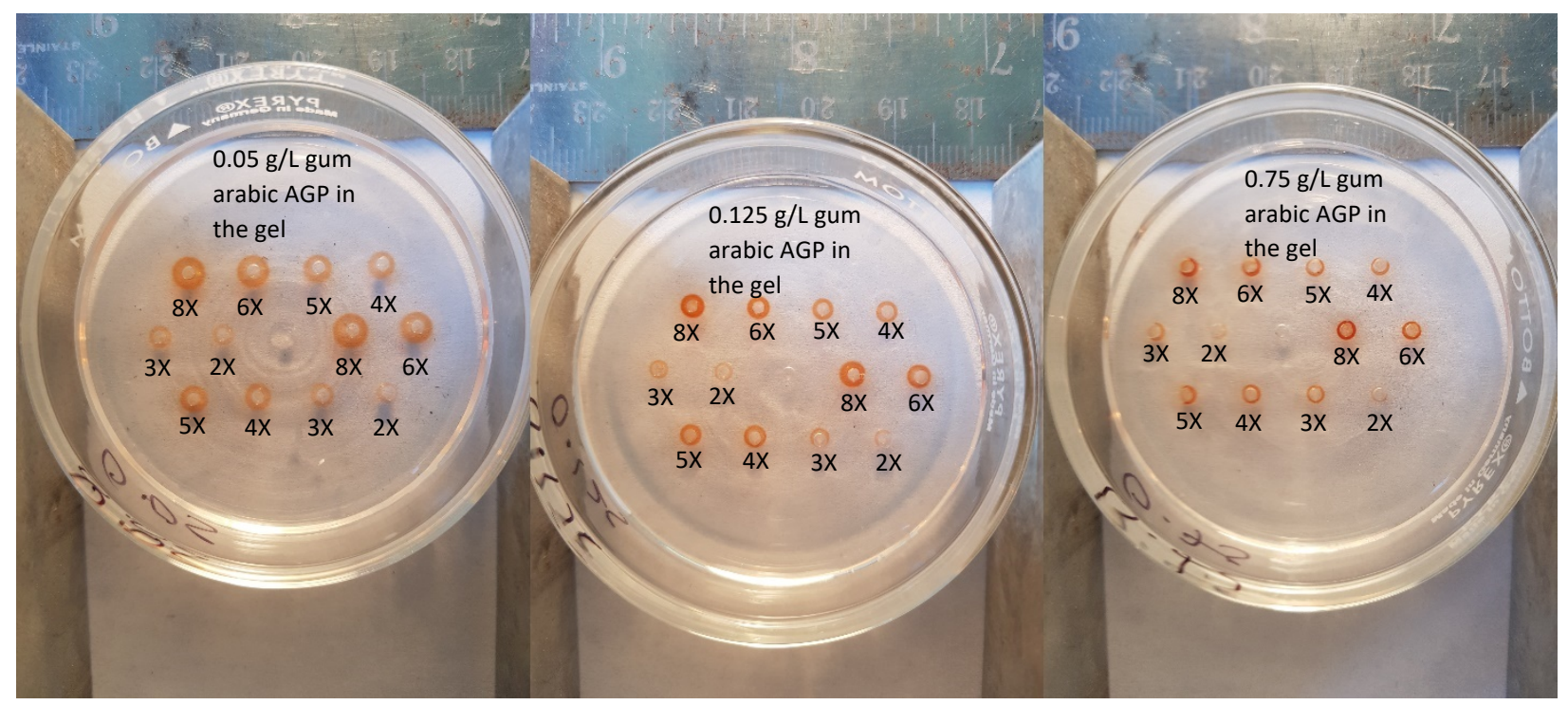

Figure S9. 'Reverse' gel assay optimization process; $\beta$ DGlc concentrations ranging from $2 \mathrm{X}$ to $8 \mathrm{X}$ where $\mathrm{X}=0.13 \mathrm{mM}$ 

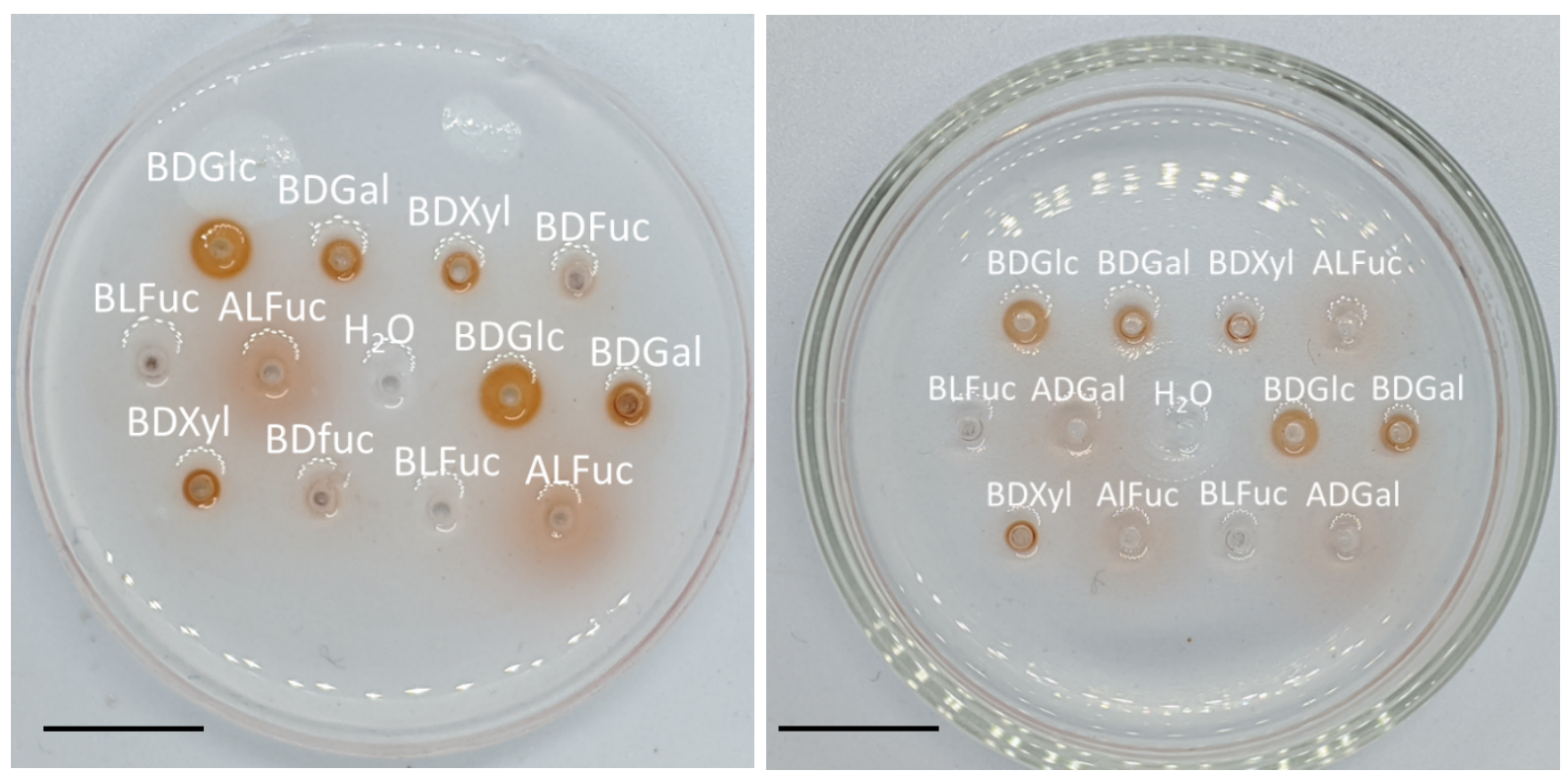

Figure S10. Reverse gel assay with AGP in the gel and (a) Yariv reagents 1-4 and 6,7 and (b) 5 instead of 4 added to the wells. Scale bar $-1 \mathrm{~cm}$

\section{Reverse gel assay protocol:}

To create the gel mixture, a freshly prepared gum arabic solution (of appropriate concentration) is added to an Erlenmeyer flask. $10 \mathrm{~mL}$ of gel mixture per gel assay is prepared, however only 4 $\mathrm{mL}$ is required for each gel, the extra solution prevents formation of air bubbles while transferring the solution using pipette later. To the gum arabic solution, $\mathrm{NaCl}$ is added to obtain a $0.15 \mathrm{M} \mathrm{NaCl}$ solution. Agarose is then added to the gel mixture $(0.01 \mathrm{~g}$ per $1 \mathrm{~mL}$ of gel mixture). A watch glass is placed on top of the Erlenmeyer flask (to prevent evaporation), following which the Erlenmeyer flask is microwaved until the contents are boiling ( $\sim 10$ seconds for a $10 \mathrm{~mL}$ mixture). Immediately after, $0.02 \% \mathrm{w} / \mathrm{v} \mathrm{NaN}(0.01 \mathrm{~mL}$ per $1 \mathrm{~mL}$ of gel mixture) was added to the flask. The solution is then dispensed into petri dishes using a p1000 pipet $(4 \mathrm{~mL}$ of gel mixture for each gel). Once the solution was added to each dish, a custom-made well mold device was inserted into each dish to create the wells in which Yariv reagents are to be added. The well molds were removed after the gels set for 10 minutes, leaving a gel with 13 wells that could be used to house the Yariv reagent. The appropriate Yariv reagent was then inserted into each well using a $10 \mu \mathrm{L}$ Hamilton Microliter ${ }^{\mathrm{TM}}$ PGC syringe; $1 \mu \mathrm{l}$ of $1.04 \mathrm{mM}$ Yariv reagent solution was added into each well. The gels were placed on a platform in a large basin filled with water and covered with foil, to create a moist environment that would prevent the gels from drying out overnight. The gels were left for approximately 16 hours before imaging the next day. 


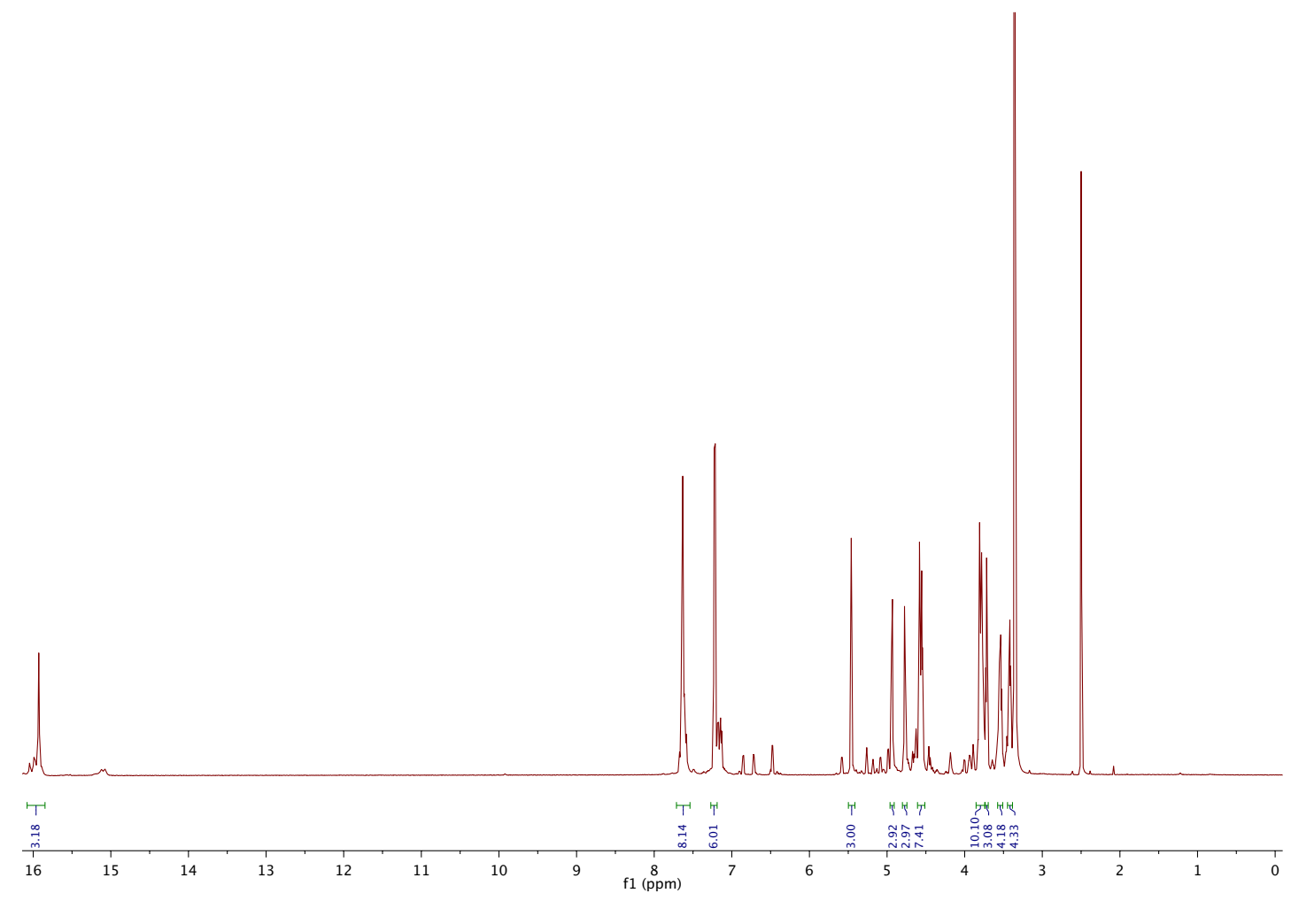

$\alpha$-Gal Yariv $5-{ }^{1} \mathrm{H}$ NMR

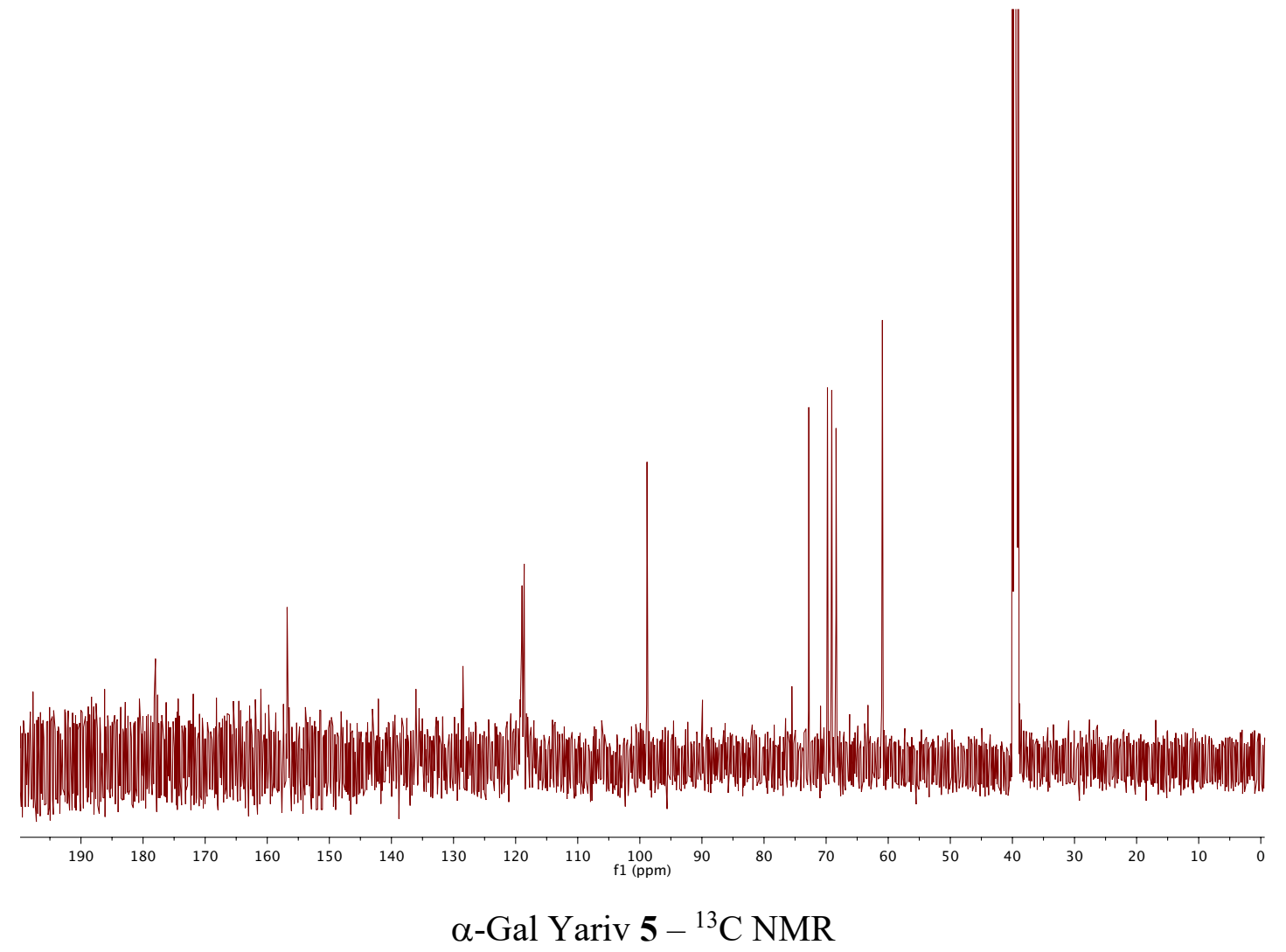




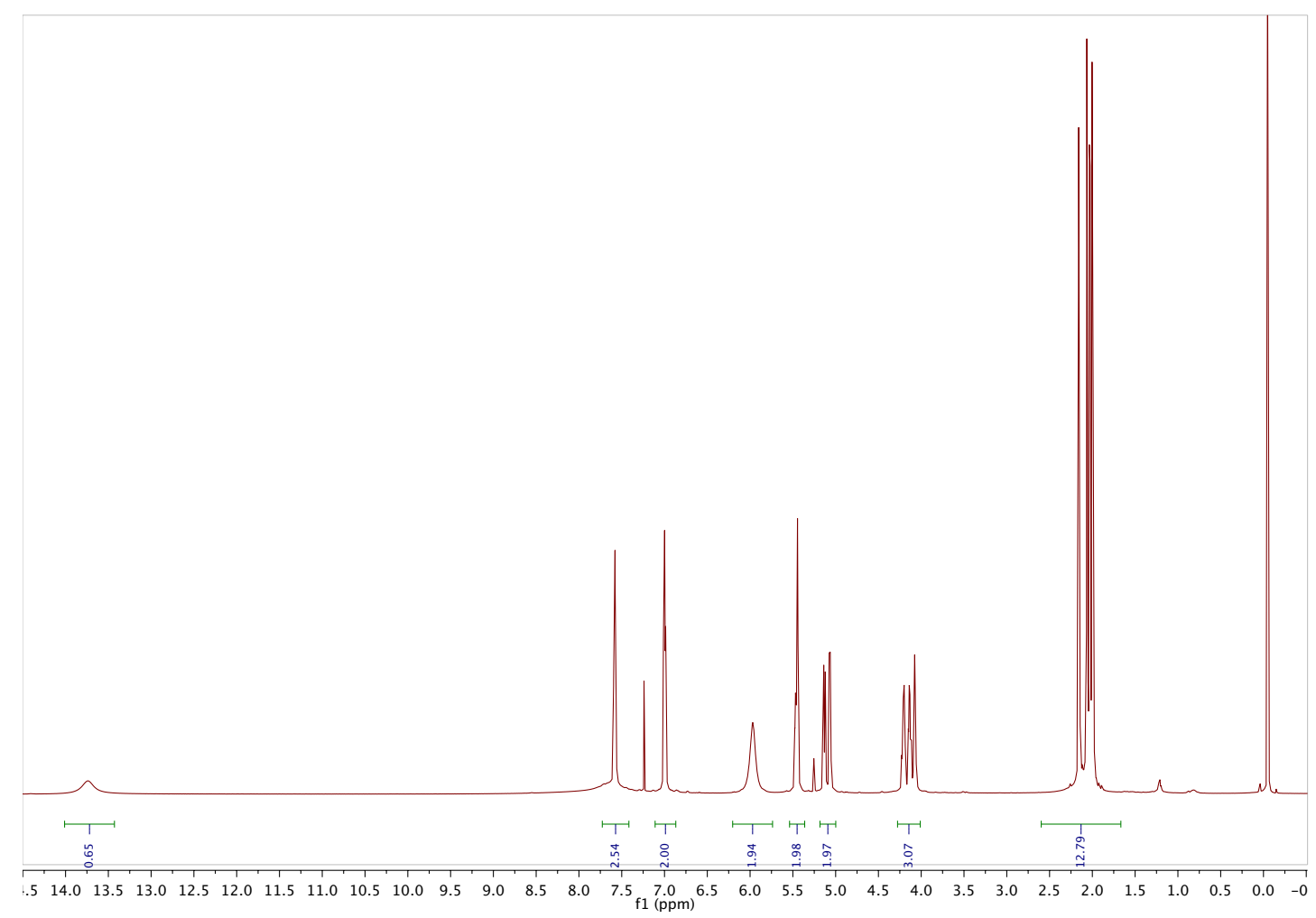

Tetraacetyl mono-galactosyl Yariv S1 $-{ }^{1} \mathrm{H}$ NMR

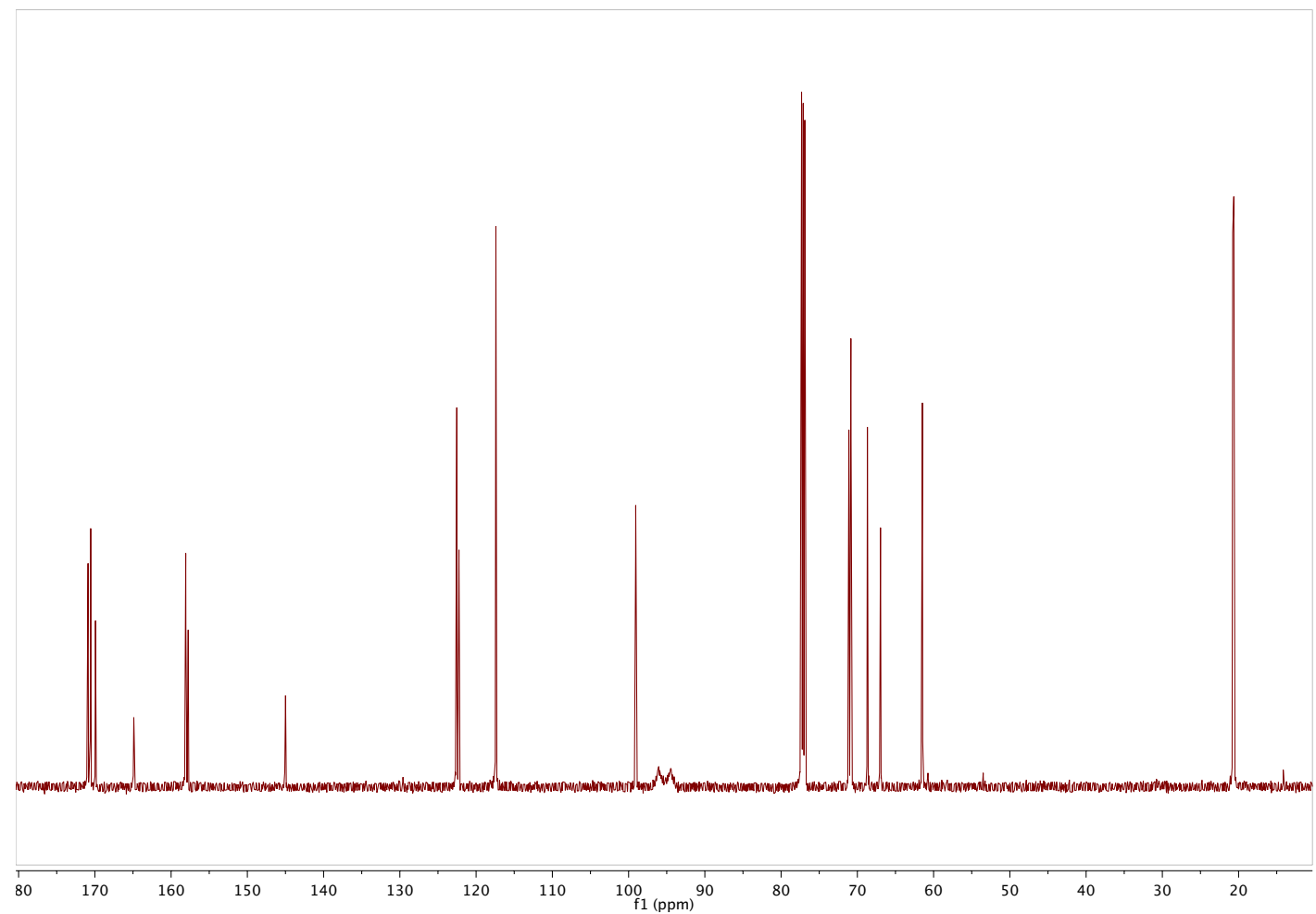

Tetraacetyl mono-galactosyl Yariv S1 $-{ }^{13} \mathrm{C}$ NMR 


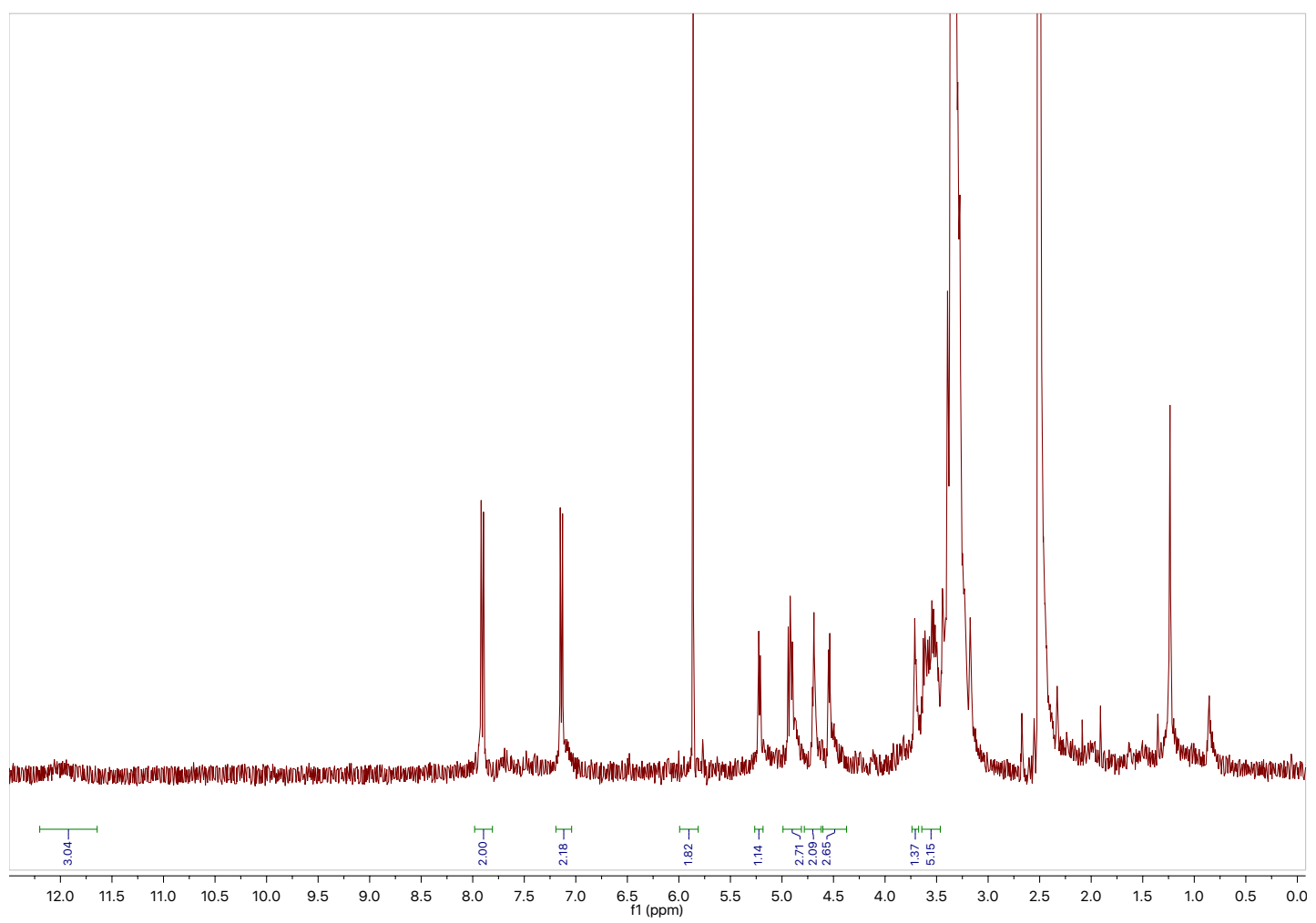

Mono-galactosyl Yariv S2 $-{ }^{1} \mathrm{H}$ NMR (DMSO $d_{6}$ )

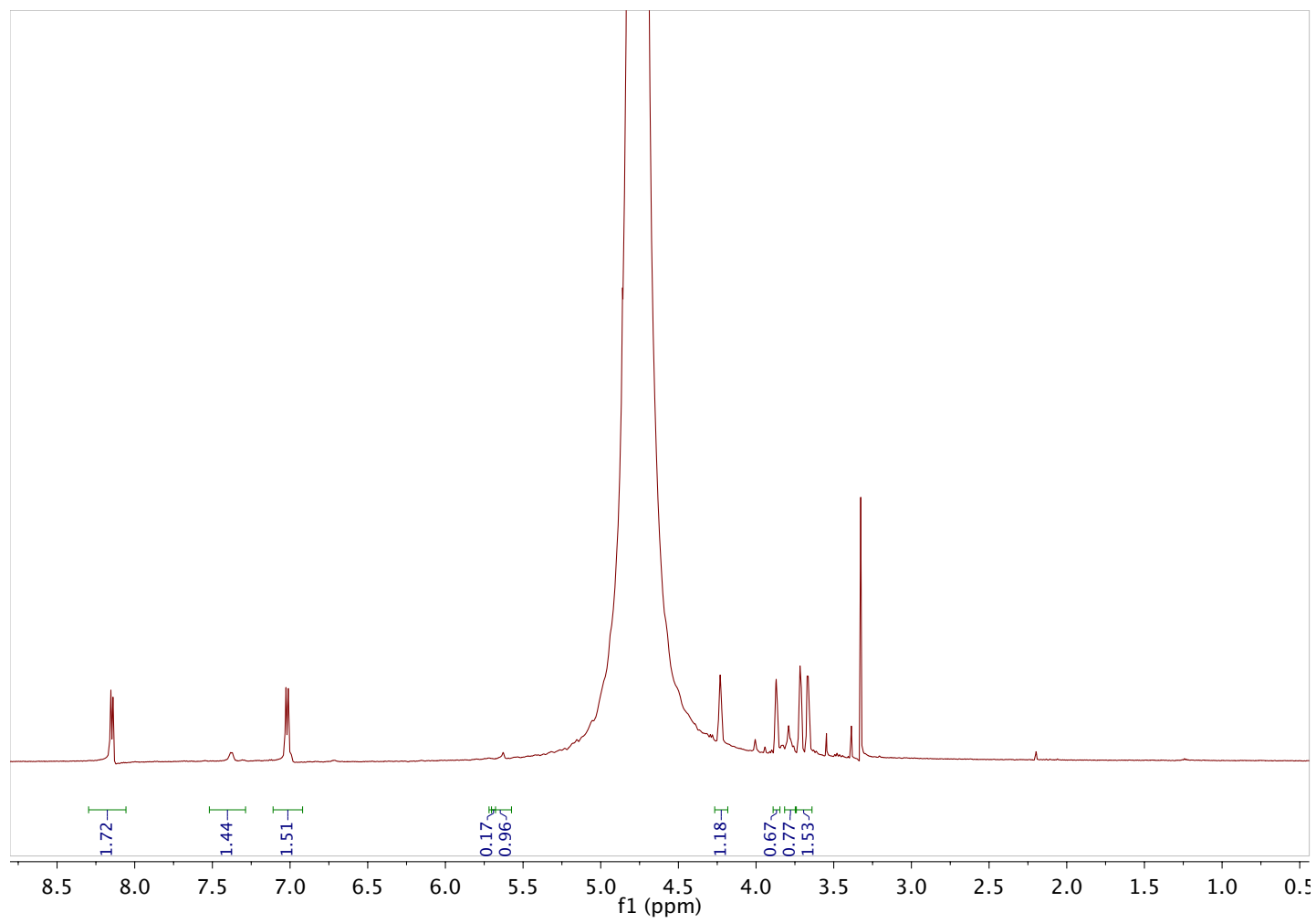

Mono-galactosyl Yariv S2 - ${ }^{1} \mathrm{H}$ NMR $\left(\mathrm{D}_{2} \mathrm{O}\right)$ 